Sci. China Math. 63 (2020), no. 3, 501-520.

\title{
UNIVERSAL SUMS OF THREE QUADRATIC POLYNOMIALS
}

\author{
ZHI-WEI SUN \\ Department of Mathematics, Nanjing University \\ Nanjing 210093, People's Republic of China \\ zwsun@nju.edu.cn \\ http://maths.nju.edu.cn/ $\sim$ zwsun
}

\begin{abstract}
Let $a, b, c, d, e, f$ be integers with $a \geqslant c \geqslant e>0, b>-a$ and $b \equiv a(\bmod 2)$, $d>-c$ and $d \equiv c(\bmod 2), f>-e$ and $f \equiv e(\bmod 2)$. Suppose that $b \geqslant d$ if $a=c$, and $d \geqslant f$ if $c=e$. When $b(a-b), d(c-d)$ and $f(e-f)$ are not all zero, we prove that if each $n \in \mathbb{N}=\{0,1,2, \ldots\}$ can be written $x(a x+b) / 2+y(c y+d) / 2+z(e z+f) / 2$ with $x, y, z \in \mathbb{N}$ then the tuple $(a, b, c, d, e, f)$ must be on our list of 473 candidates, and show that 56 of them meet our purpose. When $b \in[0, a), d \in[0, c)$ and $f \in[0, e)$, we investigate the universal tuples $(a, b, c, d, e, f)$ over $\mathbb{Z}$ for which any $n \in \mathbb{N}$ can be written $x(a x+b) / 2+y(c y+d) / 2+z(e z+f) / 2$ with $x, y, z \in \mathbb{Z}$, and show that there are totally 12082 such candidates some of which are proved to be universal tuples over $\mathbb{Z}$. For example, we show that any $n \in \mathbb{N}$ can be written as $x(x+1) / 2+y(3 y+1) / 2+z(5 z+1) / 2$ with $x, y, z \in \mathbb{Z}$, and conjecture that each $n \in \mathbb{N}$ can be written as $x(x+1) / 2+y(3 y+1) / 2+z(5 z+1) / 2$ with $x, y, z \in \mathbb{N}$.
\end{abstract}

\section{INTRODUCTION}

Recall that the triangular numbers have the form $T_{x}=x(x+1) / 2$ with $x \in \mathbb{Z}$. Since $T_{-1-n}=T_{n}$ for all $n \in \mathbb{N}=\{0,1,2, \ldots\}$, we have $\left\{T_{x}: x \in \mathbb{Z}\right\}=\left\{T_{n}: n \in \mathbb{N}\right\}$. In 1796 Gauss proved Fermat's assertion that each $n \in \mathbb{N}$ can be written as the sum of three triangular numbers. For each $m=3,4,5, \ldots$ the $m$-gonal numbers (or polygonal numbers of order $m$ ) are given by

$$
p_{m}(x):=(m-2)\left(\begin{array}{l}
x \\
2
\end{array}\right)+x=\frac{x((m-2) x-(m-4))}{2} \quad(x \in \mathbb{N}) .
$$

Note that $p_{3}(x)=T_{x}$ and $p_{4}(x)=x^{2}$. For $m \in\{5,6,7, \ldots\}$, those $p_{m}(x)$ with $x \in \mathbb{Z}$ are called generalized $m$-gonal numbers.

For a subset $S$ of $\mathbb{Z}$ and polynomials $f_{1}(x), f_{2}(x), f_{3}(x)$ with $f_{i}(S)=\left\{f_{i}(x): x \in\right.$ $S\} \subseteq \mathbb{N}$ for $i=1,2,3$, if any $n \in \mathbb{N}$ can be written as $f_{1}(x)+f_{2}(y)+f_{3}(z)$ with $x, y, z \in S$ then we call the sum $f_{1}(x)+f_{2}(y)+f_{3}(z)$ universal over $S$.

2010 Mathematics Subject Classification. Primary 11E25; Secondary 11D85, 11E20.

Keywords. Representations of integers, universal sums, quadratic polynomials.

Supported by the National Natural Science Foundation of China (grant 11571162) and the NSFCRFBR Cooperation and Exchange Program (grant 11811530072). 
Let $\mathbb{Z}^{+}=\{1,2,3, \ldots\}$. In 1862 Liouville (cf. Berndt [B, p. 82] and Dickson [D99, p. 23]) determined all those universal sums $a T_{x}+b T_{y}+c T_{z}$ (over $\mathbb{N}$ or $\mathbb{Z}$ ) with $a, b, c \in \mathbb{Z}^{+}$. It is known that $a x^{2}+b y^{2}+c z^{2}$ is not universal (over $\mathbb{N}$ or $\mathbb{Z}$ ) for any $a, b, c \in \mathbb{Z}^{+}$(cf. $[\mathrm{DW}])$. The determination of those universal sums $a p_{i}(x)+b p_{j}(y)+c p_{k}(z)$ (over $\mathbb{N}$ or $\mathbb{Z}$ ) with $\{i, j, k\}=\{3,4\}$ and $a, b, c \in \mathbb{Z}^{+}$was proposed by the author [S07] and completed via the three papers [S07], [GPS] and [OS]. Note that

$$
\left\{T_{x}+T_{y}: x, y \in \mathbb{Z}\right\}=\left\{x^{2}+2 T_{y}: x, y \in \mathbb{Z}\right\}
$$

as observed by Euler (cf. [D99, p. 11]); in fact, $x^{2}+2 T_{y}=T_{x+y}+T_{y-x}=T_{x+y}+T_{x-y-1}$.

The author [S15] showed that there are only 95 candidates for universal sums $a p_{i}(x)+$ $b p_{j}(y)+c p_{k}(z)$ over $\mathbb{N}$ with $a, b, c \in \mathbb{Z}^{+}, i, j, k \in\{3,4,5, \ldots\}$ and $\max \{i, j, k\} \geqslant 5$. Though none of the 95 sums has been proved to be universal over $\mathbb{N}$, many of them have been proved to be universal over $\mathbb{Z}$ (cf. [S15] and [JOS]).

For $c \in \mathbb{Z}^{+}$and $m \in\{3,4, \ldots\}$, clearly $c p_{m}(x)=x\left(a_{0} x+b_{0}\right) / 2$ with $a_{0}=c(m-2)$ and $b_{0}=-c(m-4) \in\left(-a_{0}, a_{0}\right]$. Instead of $c p_{m}(x)$, we may consider more general polynomials

$$
\psi_{a, b}(x):=\frac{x(a x+b)}{2} \text { with } a \in \mathbb{Z}^{+}, b \in \mathbb{Z}, b>-a \text { and } a \equiv b \quad(\bmod 2) .
$$

Clearly, $\psi_{a, b}(\mathbb{N}) \subseteq \mathbb{N}$. For positive integers $a, c, e$ and integers $b>-a, d>-c, f>-e$ with $a+b, c+d, e+f$ all even, if $\psi_{a, b}(x)+\psi_{c, d}(y)+\psi_{e, f}(z)$ is universal over $\mathbb{N}$ then we simply call the ordered tuple $(a, b, c, d, e, f)$ universal over $\mathbb{N}$. In view of Liouville's result (cf. [D99, p. 23]), and [S07], [GPS] and [OS], all those universal tuples $(a, b, c, d, e, f)$ over $\mathbb{N}$ with $b \in\{0, a\}, d \in\{0, c\}$ and $f \in\{0, e\}$ have been determined.

In our first theorem, we give some new universal tuples $(a, b, c, d, e, f)$ over $\mathbb{N}$ with $a|b, c| d$ and $e \mid f$.

Theorem 1.1. All the following 56 ordered tuples

$(1,3,1,1,1,1),(1,3,1,3,1,1),(1,5,1,1,1,1),(1,5,1,3,1,1),(1,7,1,1,1,1)$,

$(1,7,1,3,1,1),(1,9,1,1,1,1),(2,0,1,3,1,1),(2,0,1,3,1,3),(2,0,1,5,1,1)$,

$(2,0,1,5,1,3),(2,0,1,7,1,1),(2,0,1,7,1,3),(2,0,1,9,1,1),(2,0,1,9,1,3)$,

$(2,0,1,11,1,1),(2,0,1,11,1,3),(2,0,1,13,1,1),(2,0,1,13,1,3),(2,0,1,15,1,1)$,

$(2,0,2,0,1,3),(2,2,1,3,1,1),(2,2,1,5,1,1),(2,2,1,7,1,1),(2,2,2,0,1,3)$,

$(2,2,2,0,1,5),(2,2,2,0,1,7),(2,2,2,0,1,9),(2,4,1,1,1,1),(2,4,2,0,1,1)$,

$(2,4,2,0,1,3),(2,4,2,2,1,1),(2,4,2,2,2,0),(2,6,1,1,1,1),(2,6,1,3,1,1)$,

$(2,6,2,0,1,1),(2,6,2,0,1,3),(2,6,2,2,1,1),(2,6,2,2,2,0),(2,8,1,1,1,1)$,

$(2,8,2,0,1,1),(2,8,2,0,1,3),(2,8,2,2,2,0),(2,10,2,0,1,1),(2,10,2,0,1,3)$,

$(2,12,2,0,1,1),(2,12,2,0,1,3),(2,14,2,0,1,1),(3,3,2,0,1,3),(3,9,2,0,1,1)$,

$(3,9,2,0,1,3),(4,0,1,3,1,1),(4,0,1,5,1,1),(4,0,1,7,1,1),(4,4,1,3,1,1)$

$(8,0,1,3,1,1)$

are universal over $\mathbb{N}$.

We have the following conjecture on other possible universal tuples $(a, b, c, d, e, f)$ over $\mathbb{N}$ with $a|b, c| d$ and $e \mid f$. 
Conjecture 1.2. The following 10 ordered tuples

$(4,0,2,0,1,3),(4,0,2,0,1,5),(4,0,2,6,1,1),(4,0,2,6,2,0),(4,4,2,0,1,3)$,
$(4,8,2,0,1,1),(4,8,2,0,1,3),(4,12,2,0,1,1),(6,0,2,0,1,3),(6,6,2,0,1,3)$

are universal over $\mathbb{N}$.

Remark 1.3. It is easy to see that $(4,8,2,0,1,3)$ is universal over $\mathbb{N}$ if and only if any integer $n>2$ can be written as $x^{2}+2 y^{2}+T_{z}$ with $x \in \mathbb{N}$ and $y, z \in \mathbb{Z}^{+}$.

Now we state our second theorem.

Theorem 1.4. Let $a, c, e$ be positive integers and let $b>-a, d>-c$ and $f>-e$ be integers with $a+b, c+d, e+f$ all even. Suppose that $a \geqslant c \geqslant e$, and $b \geqslant d$ if $a=c$, and $d \geqslant f$ if $c=e$, and that the ordered tuple $(a, b, c, d, e, f)$ is universal over $\mathbb{N}$.

(i) If $a|b, c| d$ and $e \mid f$, but $b(a-b), d(c-d), f(e-f)$ are not all zero, then $(a, b, c, d, e, f)$ must be among the 56 tuples in Theorem 1.1 or the 10 tuples in Conjecture 1.1.

(ii) If $a \nmid b$ or $c \nmid d$ or $e \nmid f$, then $(a, b, c, d, e, f)$ must be among the 407 tuples listed in the Appendix.

Conjecture 1.5. All the 407 tuples in the Appendix are universal over $\mathbb{N}$. In particular,

$$
\left\{\frac{x(x+1)}{2}+\frac{y(3 y+1)}{2}+\frac{z(5 z+1)}{2}: x, y, z \in \mathbb{N}\right\}=\mathbb{N} .
$$

Remark 1.6. The author would like to offer 135 US dollars for the first proof of (1.3). In [S17] the author conjectured that any $n \in \mathbb{Z}^{+}$can be written as $x^{3}+y^{2}+T_{z}$ with $x, y \in \mathbb{N}$ and $z \in \mathbb{Z}^{+}$; we also conjecture that $x^{3}+T_{y}+z(3 z+7) / 2$ is universal over $\mathbb{N}$.

Guy [G94] noted that $p_{5}(x)+p_{5}(y)+p_{5}(z)$ is universal over $\mathbb{Z}$. Sun [S15] proved that if $a p_{5}(x)+b p_{5}(y)+c p_{5}(z)$ is universal over $\mathbb{Z}$ with $a, b, c \in \mathbb{Z}^{+}$and $a \leqslant b \leqslant c$ then $(a, b, c)$ is among the following 20 triples:

$$
(1,1, i)(i=1, \ldots, 6,8,9,10),(1,2, j)(j=2,3,4,6,8),(1,3, k)(k=3,4,6,7,8,9) .
$$

By Sun [S15, Theorem 1.1(ii)], Ge and Sun [GS], and Oh [O11], for each $(a, b, c)$ among the 20 triples the sum $a p_{5}(x)+b p_{5}(y)+c p_{5}(z)$ is indeed universal over $\mathbb{Z}$.

Recall that $T_{x}+T_{y}+T_{z}$ is universal over $\mathbb{Z}$. It is easy to see that

$$
\psi_{1,1}(\mathbb{Z})=\left\{T_{x}: x \in \mathbb{Z}\right\}=\{x(2 x+1): x \in \mathbb{Z}\}=\psi_{4,2}(\mathbb{Z}) .
$$

Motivated by this, Sun [S17] investigated universal sums over $\mathbb{Z}$ of the form $x(a x+1)+$ $y(b y+1)+z(c z+1)$ with $1 \leqslant a \leqslant b \leqslant c$, or the form $x(a x+b)+y(a y+c)+z(a z+d)$ with $2<a \geqslant b \geqslant c \geqslant d \geqslant 0$. Later, Ju and $\mathrm{Oh}[\mathrm{JO}]$ proved some conjectures of Sun [S17] in this direction.

For $a \in \mathbb{Z}^{+}$, clearly $\psi_{a,-b}(\mathbb{Z})=\psi_{a, b}(\mathbb{Z})$ for all $b=0, \ldots, a$ with $b \equiv a(\bmod 2)$, and $\psi_{a, a}(\mathbb{Z})=\psi_{4 a, 2 a}(\mathbb{Z})$ by $(1.4)$. Thus we are led to find all the sums

$$
\psi_{a, b}(x)+\psi_{c, d}(y)+\psi_{e, f}(z)=\frac{x(a x+b)}{2}+\frac{y(c y+d)}{2}+\frac{z(e z+f)}{2}
$$

which are universal over $\mathbb{Z}$, where $a, c, e \in \mathbb{Z}^{+}, b, d, f \in \mathbb{N}, b<a$ and $a \equiv b(\bmod 2)$, $d<c$ and $c \equiv d(\bmod 2)$, and $f<e$ and $e \equiv f(\bmod 2)$. If the sum in $(1.5)$ is universal over $\mathbb{Z}$, then we say that the ordered tuple $(a, b, c, d, e, f)$ is universal over $\mathbb{Z}$. 
Theorem 1.7. Let $a, b, c, d, e, f \in \mathbb{N}$ with $a>b, c>d, e>f, a \equiv b(\bmod 2), c \equiv d$ $(\bmod 2), e \equiv f(\bmod 2), a \geqslant c \geqslant e \geqslant 2$, and $b \geqslant d$ if $a=c$, and $d \geqslant f$ if $c=e$. Suppose that the ordered tuple $(a, b, c, d, e, f)$ is universal over $\mathbb{Z}$. Then $(a, b, c, d, e, f)$ must be among the 12082 ordered tuples listed in [S17a].

Remark 1.8. Chan and $\mathrm{Oh}[\mathrm{CO}]$ showed that there are only finitely many equivalence classes of positive ternary universal integral quadratic polynomials. We have analysed those tuples $(a, b, c, d, e, f)$ with $a \leqslant 5$ listed in [S17a], only the following 10 tuples

$$
\begin{aligned}
& (5,1,2,0,2,0),(5,3,2,0,2,0),(5,1,4,0,2,0),(5,3,4,0,2,0),(5,1,4,0,3,1), \\
& (5,3,4,0,3,1),(5,1,5,1,2,0),(5,3,5,3,2,0),(5,3,4,0,4,0),(5,3,5,3,4,0)
\end{aligned}
$$

have not yet been proved to be universal over $\mathbb{Z}$.

For polynomials $f_{1}, f_{2}, f_{3}, f_{4}$ with $f_{i}(\mathbb{Z}) \subseteq \mathbb{N}$, if

$$
\left\{f_{1}(x)+f_{2}(y): x, y \in \mathbb{Z}\right\}=\left\{f_{3}(x)+f_{4}(y): x, y \in \mathbb{Z}\right\}
$$

then we say that $f_{1}(x)+f_{2}(y)$ is equivalent to $f_{3}(x)+f_{4}(y)$ and write $f_{1}(x)+f_{2}(y) \sim$ $f_{3}(x)+f_{4}(y)$ for this. (1.1) indicates that $T_{x}+T_{y} \sim x^{2}+2 T_{y}$. In light of this, we obtain the following auxiliary result which has its own interest.

Theorem 1.9. (i) For any $a \in \mathbb{Z}^{+}$and $b \in \mathbb{N}$ with $b \leqslant a / 2$, we have

$$
x(a x+b)+y(a y+a-b) \sim a T_{x}+\psi_{a, a-2 b}(y) .
$$

(ii) We have

$$
\begin{aligned}
x^{2}+T_{y} & \sim p_{5}(x)+2 p_{5}(y), \\
T_{x}+2 T_{y} & \sim p_{5}(x)+p_{8}(y), \\
x^{2}+4 T_{y} & \sim 4 p_{5}(x)+p_{8}(y), \\
T_{x}+T_{y} & \sim \psi_{5,1}(x)+\psi_{5,3}(y) .
\end{aligned}
$$

Remark 1.10. (1.6) with $a=1$ and $b=0$ gives (1.1). Putting $a=3$ and $b=1$ in (1.6) we get

$$
2 p_{5}(x)+p_{8}(y) \sim 3 T_{x}+p_{5}(y) .
$$

With helps of Theorem 1.9 and the theory of ternary quadratic forms, we establish the following new result.

Theorem 1.11. (i) We have

$$
\left\{\frac{x(x+1)}{2}+\frac{y(3 y+1)}{2}+\frac{z(5 z+1)}{2}: x, y, z \in \mathbb{Z}\right\}=\mathbb{N} .
$$

(ii) For any $\delta \in\{0,1\}$ and $r \in\{1,3,5\}$, we have

$$
\left\{x(x+\delta)+\frac{y(3 y+1)}{2}+\frac{z(5 z+r)}{2}: x, y, z \in \mathbb{Z}\right\}=\mathbb{N} .
$$


(iii) For any $r, s, t \in\{1,3\}$ with $\{r, s\} \neq\{3\}$, we have

$$
\left\{\frac{x(3 x+r)}{2}+\frac{y(3 y+s)}{2}+\frac{z(5 z+t)}{2}: x, y, z \in \mathbb{Z}\right\}=\mathbb{N} \text {. }
$$

(iv) Let $s, t \in\{1,3,5\}$ with $\{s, t\} \neq\{5\}$. Then

$$
\left\{\frac{x(3 x+1)}{2}+\frac{y(5 y+s)}{2}+\frac{z(5 z+t)}{2}: x, y, z \in \mathbb{Z}\right\}=\mathbb{N} \text {. }
$$

Remark 1.12. The author [S17, Conjecture 1.2] conjectured that $x^{2}+y(3 y+1) / 2+$ $z(5 z+3) / 2$ is universal over $\mathbb{N}$. In view of (1.11), and (1.14) with $r=3, s=1$ and $t \in\{1,3\}$, the tuples $(6,4,6,2,5,1)$ and $(6,4,6,2,5,3)$ are universal over $\mathbb{Z}$.

We are going to prove Theorem 1.1 in Section 3 based on some lemmas given in the next section. Theorems 1.4 and 1.7 and Theorems 1.9 and 1.11 will be shown in Sections 4 and 5 respectively.

\section{Some LEMMAS}

The Gauss-Legendre theorem on sums of three squares (cf. [N96, pp. 17-23]) asserts that $\left\{x^{2}+y^{2}+z^{2}: x, y, z \in \mathbb{Z}\right\}=\mathbb{N} \backslash\left\{4^{k}(8 l+7): k, l \in \mathbb{N}\right\}$. For $n \in \mathbb{N}$ we define

$$
R_{3}(n):=\mid\left\{(x, y, z) \in \mathbb{Z}^{3}: x^{2}+y^{2}+z^{2}=n \text { and } \operatorname{gcd}(x, y, z)=1\right\} \mid .
$$

Lemma 2.1 (Gauss). Let $n \in \mathbb{N}$. Then $R_{3}(1)=6, R_{3}(2)=12, R_{3}(3)=8$, and

$$
R_{3}(n)= \begin{cases}12 h(-n) & \text { if } n>3 \text { and } n \equiv 1,2(\bmod 4) \\ 24 h(-n) & \text { if } n>3 \text { and } n \equiv 3(\bmod 8) \\ 0 & \text { if } 4 \mid n \text { or } n \equiv 7(\bmod 8)\end{cases}
$$

where $h(-n)$ denotes the class number of the field $\mathbb{Q}(\sqrt{-n})$.

Remark 2.2. One may consult [P, p. 140] for this classical result.

Lemma 2.3. Let $n \in \mathbb{Z}^{+}$be squarefree.

(i) $h(-n)=1$ if and only if $n$ is among the following nine numbers

$1,2,3,7,11,19,43,67,163$.

(ii) $h(-n)=2$ if and only if $n$ is among the following numbers

$5,6,10,13,15,22,35,37,51,58,91,115,123,187,235,267,403,427$.

(iii) $h(-n)=3$ if and only if $n$ is among the following numbers

23, 31, 59, 83, 107, 139, 211, 283, 307, 331, 379, 499, 547, 643, 883, 907. 
(iv) $h(-n)=4$ if and only if $n$ is among the following numbers

$14,17,21,30,33,34,39,42,46,55,57,70,73,78,82,85,93,97$, $102,130,133,142,155,177,190,193,195,203,219,253,259,291$, $323,355,435,483,555,595,627,667,715,723,763,795,955,1003$, 1027, 1227, 1243, 1387, 1411, 1435, 1507, 1555.

(v) If $n \equiv 1(\bmod 8)$, then $h(-n) \in\{5,6,7,8\}$ if and only if $n$ is among the following numbers

41, 65, 105, 113, 137, 145, 217, 265, 273, 313, 337, 345, 385, 457, 505, 553, 697, 793.

Remark 2.4. This is a known result, see, [A], [ARW], [W], [Wa] and [We].

Lemma 2.5. Any integer $n>1$ can be written as $T_{x}+T_{y}+T_{z}$ with $x, y \in \mathbb{Z}^{+}$and $z \in \mathbb{N}$.

Proof. By Gauss' result, $n=T_{x}+T_{y}+T_{z}$ for some $x, y, z \in \mathbb{N}$ with $x \geqslant y \geqslant z$. If $y>0$ then $x, y \in \mathbb{Z}^{+}$. If $y=0$, then $z$ is also zero and $n=T_{x}$ is a triangular number.

Now assume that $n=T_{m}>1$ with $m \in \mathbb{N}$. Clearly, $m>1$. If $8 T_{m}+3=(2 m+1)^{2}+2$ is divisible by $d^{2}$ for some integer $d>1$, then

$$
\left|\left\{(x, y, z) \in \mathbb{Z}^{3}: x^{2}+y^{2}+z^{2}=8 T_{m}+3 \& \operatorname{gcd}(x, y, z)=d\right\}\right|=R_{3}\left(\frac{8 T_{m}+3}{d^{2}}\right)>0
$$

by Lemma 2.1, and hence $8 T_{m}+3=x^{2}+y^{2}+z^{2}$ for some positive odd integers $x, y, z$ with $\min \{x, y\} \geqslant d>1$. When $8 T_{m}+3=(2 m+1)^{2}+2$ is squarefree, by Lemma 2.1 and Lemma 2.3(i) we have $R_{3}\left(8 T_{m}+3\right)=24 h\left(-8 T_{m}-3\right)>24$ since $8 T_{m}+3=(2 m+1)^{2}+2 \notin\{1,2,3,7,11,19,43,67,163\}$, hence there are integral solutions of the equation $x^{2}+y^{2}+z^{2}=8 T_{m}+3$ other than the 24 trivial solutions

$$
( \pm 1, \pm 1, \pm(2 m+1)),( \pm 1, \pm(2 m+1), \pm 1),( \pm(2 m+1), \pm 1, \pm 1) .
$$

Thus, for some $x, y \in \mathbb{Z}^{+}$and $z \in \mathbb{N}$ we have

$$
8 T_{m}+3=(2 x+1)^{2}+(2 y+1)^{2}+(2 z+1)^{2} \text {, i.e., } T_{m}=T_{x}+T_{y}+T_{z} .
$$

This concludes our proof.

Lemma 2.6. Let $n>2$ be an integer. Then

$$
4 n+2=x^{2}+y^{2}+z^{2} \quad \text { for some } x, y, z \in \mathbb{N} \text { with } x \geqslant y>1 \text {. }
$$

Proof. If $d^{2} \mid 4 n+2$ for some integer $d>1$, then $R_{3}\left((4 n+2) / d^{2}\right)>0$ (by Lemma 2.1 ) and hence $4 n+2=(d x)^{2}+(d y)^{2}+(d z)^{2}$ for some $x, y, z \in \mathbb{N}$ with two of $d x, d y, d z$ odd and greater than one. If neither $4 n$ nor $4 n+1$ is a square, then $4 n+2$ is not of the 
form $x^{2}+2$ or $x^{2}+1$ with $x \in \mathbb{Z}$, and by the Gauss-Legendre theorem we can write $4 n+2=x^{2}+y^{2}+z^{2}$ with $x, y, z \in \mathbb{N}$ and $x \geqslant y>1$.

Now assume that $4 n+\delta=t^{2}$ with $\delta \in\{0,1\}$ and $t \in \mathbb{N}$, and that $4 n+2$ is squarefree. Then $R_{3}(4 n+2)=12 h(-4 n-2)>24$ by Lemmas 2.1 and 2.2. (Note that none of $22-2,22-1,58-2,58-1$ is a square.) So the equation $4 n+2=x^{2}+y^{2}+z^{2}$ has integral solutions other than the trivial solutions

$$
( \pm 1, \pm \bar{\delta}, \pm t),( \pm \bar{\delta}, \pm 1, \pm t),( \pm 1, \pm t, \pm \bar{\delta}),( \pm \bar{\delta}, \pm t, \pm 1),( \pm t, \pm 1, \pm \bar{\delta}), \quad( \pm t, \pm \bar{\delta}, \pm 1)
$$

where $\bar{\delta}=1-\delta$. (No matter $\delta=0$ or 1 there are exactly 24 trivial solutions.) Hence $4 n+2=x^{2}+y^{2}+z^{2}$ for some $x, y, z \in \mathbb{N}$ with $x \geqslant y \geqslant z$ and $y>1$. This ends the proof.

Lemma 2.7. Let $n>1$ be an odd integer. Then, for each $m=1,2$ there are $x, y, z \in \mathbb{N}$ with $\max \{x, z\}>0$ and $\max \{y, z\}>0$ such that $x^{2}+y^{2}+m z^{2}=n^{2}$.

Proof. In 1907 Hurwitz (cf. [D99, p. 271]) showed that

$$
\left|\left\{(x, y, z) \in \mathbb{Z}^{3}: x^{2}+y^{2}+z^{2}=n^{2}\right\}\right|=6 \prod_{p \mid n}\left(p^{\operatorname{ord}_{p}(n)}+\left(1-\left(\frac{-1}{p}\right)\right) \frac{p^{\operatorname{ord}_{p}(n)}-1}{p-1}\right),
$$

where $\operatorname{ord}_{p}(n)$ is the order of $n$ at the prime $p$ and $(\dot{p})$ is the Legendre symbol. This implies that

$$
\left|\left\{(x, y, z) \in \mathbb{Z}^{3}: x^{2}+y^{2}+z^{2}=n^{2}\right\}\right| \geqslant 6 \prod_{p \mid n} p^{\operatorname{ord}_{p}(n)}=6 n>6 .
$$

So the equation $x^{2}+y^{2}+z^{2}=n^{2}$ has integral solutions other than the 6 trivial solutions $(0,0, \pm n),(0, \pm n, 0),( \pm n, 0,0)$. This proves the desired result in the case $m=1$.

Now we consider the case $m=2$. By a result of Cooper and Lam [CL], we have

$$
\begin{aligned}
\left|\left\{(x, y, z) \in \mathbb{Z}^{3}: x^{2}+y^{2}+2 z^{2}=n^{2}\right\}\right| & =4 \prod_{p \mid n} \frac{p^{\operatorname{ord}_{p}(n)+1}-1-\left(\frac{-2}{p}\right)\left(p^{\operatorname{ord}_{p}(n)}-1\right)}{p-1} \\
& \geqslant 4 \prod_{p \mid n} \frac{p^{\operatorname{ord}_{p}(n)+1}-p^{\operatorname{ord}_{p}(n)}}{p-1}=4 n>4 .
\end{aligned}
$$

So the equation $x^{2}+y^{2}+2 z^{2}=n^{2}$ has integral solutions other than the 4 trivial solutions $( \pm n, 0,0)$ and $(0, \pm n, 0)$. This proves the desired result in the case $m=2$.

Lemma 2.8. Let $m>3$ be an integer. Then we can write $T_{m}=x^{2}+y^{2}+T_{z}$ with $x, y, z \in \mathbb{N}, y \geqslant 3$ and $z \geqslant 1$.

Proof. It is easy to verify the desired result for $m=4,5,6,7,8$. When $m=a^{2}$ with $a \in\{3,4, \ldots\}$, the desired result also holds since $T_{m}=m+T_{m-1}=0^{2}+a^{2}+T_{a^{2}-1}$.

Now we assume that $m$ is greater than 8 and not a square. By Dickson [D39, pp. 112$113]$,

$$
\mathbb{N} \backslash\left\{x^{2}+y^{2}+2 z^{2}: x, y, z \in \mathbb{Z}\right\}=\left\{4^{k}(16 l+14): k, l \in \mathbb{N}\right\}
$$


Thus, in view of Lemma 2.7, no matter $2 m+1$ is a square or not, $2 m+1=x^{2}+y^{2}+2 z^{2}$ for some $x, y, z \in \mathbb{N}$ with $\left(x^{2}+z^{2}\right)\left(y^{2}+z^{2}\right)>0$. Note that $x^{2}+y^{2}>1$. If $\left(x^{2}+z^{2}\right)\left(y^{2}+z^{2}\right) \leqslant$ 16 , then $x y \leqslant 4$ and $z \leqslant 2$, hence $2 m+1=x^{2}+y^{2}+2 z^{2} \leqslant 17$ which contradicts $m>8$. Thus $\left(x^{2}+z^{2}\right)\left(y^{2}+z^{2}\right)>16$.

Observe that

$$
\begin{aligned}
8 T_{m}+1 & =(2 m+1)^{2}=\left(x^{2}+z^{2}+\left(y^{2}+z^{2}\right)\right)^{2} \\
& =\left(x^{2}+z^{2}-\left(y^{2}+z^{2}\right)\right)^{2}+4\left(x^{2}+z^{2}\right)\left(y^{2}+z^{2}\right) \\
& =\left(x^{2}-y^{2}\right)^{2}+4\left(\left(x y+z^{2}\right)^{2}+(x z-y z)^{2}\right) .
\end{aligned}
$$

Since $x \not \equiv y(\bmod 2)$ and $\left|x^{2}-y^{2}\right| \geqslant x+y>1$, we have $\left|x^{2}-y^{2}\right|=2 w+1$ for some $w \in \mathbb{Z}^{+}$. Note that $\left(x y+z^{2}\right)^{2}+(|x-y| z)^{2}=\left(x^{2}+z^{2}\right)\left(y^{2}+z^{2}\right) \equiv 0(\bmod 2)$. Thus

$$
u:=\frac{x y+z^{2}+|x-y| z}{2} \in \mathbb{N} \text { and } v:=\left|\frac{x y+z^{2}-|x-y| z}{2}\right| \in \mathbb{N} .
$$

Since

$$
u^{2}+v^{2}=\frac{\left(x y+z^{2}\right)^{2}+(x z-y z)^{2}}{2}=\frac{\left(x^{2}+z^{2}\right)\left(y^{2}+z^{2}\right)}{2}>8,
$$

we have $\max \{u, v\} \geqslant 3$. Finally, $8 T_{m}+1=(2 w+1)^{2}+8\left(u^{2}+v^{2}\right)$ and hence $T_{m}=$ $u^{2}+v^{2}+T_{w}$. This concludes the proof.

\section{Proof of Theorem 1.1}

Proof of Theorem 1.1. We first make a useful observation:

$$
\frac{z(z+2 k+1)}{2}=T_{z+k}-T_{k} \text { for all } k \in \mathbb{N}
$$

(a) Let $k \in\{1,2,3\}$. Clearly $(1,2 k+1,1,3,1,1)$ is universal over $\mathbb{N}$ if and only if each integer $n \geqslant T_{k}+1$ can be written as $T_{x+k}+T_{y+1}+T_{z}$ with $x, y, z \in \mathbb{N}$. Let $n$ be any integer with $n \geqslant T_{k}+1$. By Lemma 2.3, we can write $n$ as $T_{x}+T_{y}+T_{z}$ with $x, y, z \in \mathbb{N}$ and $x \geqslant y \geqslant \max \{z, 1\}$. If $x<k$, then $k \in\{2,3\}$ and $n=T_{x}+T_{y}+T_{z} \leqslant 3 T_{k-1}$. Note that $3 T_{2-1}<T_{2}+1, T_{3}+1=7$ and $3 T_{3-1}=9$. Clearly, $7=T_{3}+T_{1}+T_{0}$, $8=T_{3}+T_{1}+T_{1}$ and $9=T_{3}+T_{2}+T_{0}$. Thus $(1,2 k+1,1,3,1,1)$ is universal over $\mathbb{N}$.

Similarly, for each $k \in\{1,2,3,4\}$, the tuple $(1,2 k+1,1,1,1,1)$ is universal over $\mathbb{N}$ and so is $(2,2,2,0,1,2 k+1)$ in view of $(1.1)$.

(b) By Lemma 2.6, for any integer $n>2$ there are $x, y \in \mathbb{Z}^{+}$and $z \in \mathbb{N}$ with $z \leqslant y$ such that

$$
4 n+2=(2 x+1)^{2}+(y+z+1)^{2}+(y-z)^{2} \text {, i.e., } n=2 T_{x}+T_{y}+T_{z} \text {. }
$$

So, for any $n \in \mathbb{N}$ there are $x, y, z \in \mathbb{N}$ such that $n+3=2 T_{x+1}+T_{y+1}+T_{z}$ and hence $n=x(x+3)+y(y+3) / 2+z(z+1) / 2$. This prove the universality of $(2,6,1,3,1,1)$ over $\mathbb{N}$. As $y(y+3) / 2=T_{y+1}-1$ and $T_{y}+T_{z} \sim y^{2}+2 T_{z}$, the tuples $(2,6,1,1,1,1)$ and $(2,6,2,2,2,0)$ are also universal over $\mathbb{N}$. 
Let $k \in\{1,2,3\}$. It is easy to check that each $n=T_{k}, T_{k}+1, \ldots, k^{2}-k+6$ can be written as $2 T_{x}+T_{y}+T_{z}$ with $x, y, z \in \mathbb{N}$ and $z \geqslant k$. Let $n \in \mathbb{N}$ with $n>k(k-1)+6$. Then $n=2 T_{x}+T_{y}+T_{z}$ for some $x, y, z \in \mathbb{N}$. As $n>2 T_{2}+T_{k-1}+T_{k-1}$, either $x \geqslant 3$ or $\max \{y, z\} \geqslant k$. If $\max \{y, z\}<k$, then $x \geqslant 3 \geqslant k, T_{y}+T_{z} \leqslant 2 T_{k-1}=$ $k(k-1) \leqslant 6$ and $T_{y}+T_{z} \neq 5$. It is easy to check that $2 T_{x}+2 \delta=2 T_{\delta}+T_{x}+T_{x}$ and $2 T_{x}+2 \delta+1=2 T_{\delta}+T_{x-1}+T_{x+1}$ for $\delta=0,1 ;$ also, $2 T_{x}+4=2 T_{0}+T_{x-2}+T_{x+2}$ and $2 T_{x}+6=T_{0}+T_{3}+2 T_{x}$. Therefore $(2,2,1,2 k+1,1,1)$ is a universal tuple over $\mathbb{N}$.

In view of Lemma 2.6 , for any $n \in \mathbb{Z}^{+}$we can write

$$
8 n+6=4(2 n+1)+2=(2 x+1)^{2}+(2 y+1)^{2}+w^{2}
$$

with $x \in \mathbb{Z}^{+}$and $y, w \in \mathbb{N}$. Since $w^{2} \equiv 4(\bmod 8)$, we have $w=2(2 z+1)$ for some $z \in \mathbb{N}$. Therefore $8 n+6=(2 x+1)^{2}+(2 y+1)^{2}+4(2 z+1)^{2}$, hence $n=T_{x}+T_{y}+4 T_{z}$ and $n-1=m(m+3) / 2+T_{y}+4 T_{z}$ with $m=x-1 \in \mathbb{N}$. This proves the universality of $(4,4,1,3,1,1)$ over $\mathbb{N}$.

(c) The tuple $(2,0,1,15,1,1)$ is universal over $\mathbb{N}$ if and only if any integer $n \geqslant 28$ can be written in the form $x^{2}+T_{y}+T_{z}$ with $x, y, z \in \mathbb{N}$ and $\max \{y, z\} \geqslant 7$. It is easy to verify that every $n=28,29, \ldots, 78$ can be written as $x^{2}+T_{y}+T_{z}$ with $x, y, z \in \mathbb{N}$ and $z \geqslant 7$. Now let $n \in \mathbb{N}$ with $n>78$. We can write $n$ as $x^{2}+T_{y}+T_{z}$ with $x, y, z \in \mathbb{N}$ (cf. [S07]). Suppose that $\max \{y, z\} \leqslant 6$. Then $n-x^{2}=T_{y}+T_{z}$ belongs to the set

$R:=\left\{T_{i}+T_{j}: i, j=0, \ldots, 6\right\}=(\{0, \ldots, 31\} \cup\{36,42\}) \backslash\{5,8,14,17,19,23,26,28,29\}$

and hence $x>6$ since $36+42<n$. Note that

$$
R \subseteq \bigcup_{m=0}^{6}\left\{m^{2}, m^{2}+2, m(m+1)+0^{2}, \ldots, m(m+1)+5^{2}\right\} .
$$

Also, $x^{2}=T_{x-1}+T_{x}, x^{2}+2=T_{x-2}+T_{x+1}$ and $x^{2}+m(m+1)=T_{x-m-1}+T_{x+m}$. So the tuple $(2,0,1,15,1,1)$ is indeed universal over $\mathbb{N}$.

Let $k \in\{2,3,4,5,6\}$. By the last paragraph, any integer $n \geqslant T_{7}=28$ can be written in the form $x^{2}+T_{y}+T_{z}$ with $x, y, z \in \mathbb{N}$ and $z \geqslant 7 \geqslant k$. It is easy to verify that each $n=T_{k}, T_{k}+1, \ldots, 27$ can be written as $x^{2}+T_{y}+T_{z}$ with $x, y, z \in \mathbb{N}$ and $z \geqslant k$. Therefore the tuple $(2,0,1,2 k+1,1,1)$ is universal over $\mathbb{N}$.

(d) Let $n>1$ be an integer. We claim that the equation $4 n+1=x^{2}+y^{2}+z^{2}$ has at most $2^{3} \times 3 !=48$ integral solutions with $x-z, y-z \in\{ \pm 1\}$.

For any $y, z \in \mathbb{Z}^{+}$, it is easy to see that $2(y-1)^{2}+y^{2} \neq 2(z+1)^{2}+z^{2}$ by considering the cases $y<z, y \in\{z, z+1\}$, and $y>z+1$.

Suppose that $4 n+1=\left(z_{0}+1\right)^{2}+\left(z_{0}-1\right)^{2}+z_{0}^{2}$ for some $z_{0} \in \mathbb{N}$. Then $z_{0}>0$. For any $z \in \mathbb{N}$ with $z<z_{0}$ we have

$$
\begin{aligned}
2(z-1)^{2}+z^{2} & <(z+1)^{2}+(z-1)^{2}+z^{2}<2(z+1)^{2}+z^{2} \leqslant 2 z_{0}^{2}+\left(z_{0}-1\right)^{2} \\
& <\left(z_{0}+1\right)^{2}+\left(z_{0}-1\right)^{2}+z_{0}^{2}=4 n+1,
\end{aligned}
$$

and for any integer $z>z_{0}+1$ we have

$$
\begin{aligned}
2(z+1)^{2}+z^{2} & \geqslant(z+1)^{2}+(z-1)^{2}+z^{2} \geqslant 2(z-1)^{2}+z^{2} \geqslant 2\left(z_{0}+1\right)^{2}+\left(z_{0}+2\right)^{2} \\
& >\left(z_{0}+1\right)^{2}+\left(z_{0}-1\right)^{2}+z_{0}^{2}=4 n+1 .
\end{aligned}
$$


Note also that

$$
\begin{aligned}
2\left(z_{0}-1\right)^{2}+z_{0}^{2} & <\left(z_{0}+1\right)^{2}+\left(z_{0}-1\right)^{2}+z_{0}^{2}=4 n+1 \\
& <2 z_{0}^{2}+\left(z_{0}+1\right)^{2}<2\left(z_{0}+1\right)^{2}+z_{0}^{2}<2\left(z_{0}+2\right)^{2}+\left(z_{0}+1\right)^{2} .
\end{aligned}
$$

So $4 n+1 \neq 2(z \pm 1)^{2}+z^{2}$ for any $z \in \mathbb{N}$.

In view of the above, the claim does hold.

If $d^{2} \mid 4 n+1$ for some integer $d>1$, then $R_{3}\left((4 n+1) / d^{2}\right)>0$ by Lemma 2.1 , and hence there are $x, y, z \in \mathbb{N}$ with $\operatorname{gcd}(x, y, z)=1$ such that $4 n+1=(d x)^{2}+(d y)^{2}+(d z)^{2}$ with $d x-d y, d x-d z, d y-d z \neq \pm 1$. When $4 n+1$ is squarefree, by Lemma 2.3 we have $h(-4 n-1) \leqslant 4$ if and only if $4 n+1$ belongs to the set

$$
S:=\{13,17,21,33,37,57,73,85,93,97,133,177,193,253\} .
$$

If $4 n+1 \in S$ then we can easily write $4 n+1$ as $x^{2}+y^{2}+z^{2}$ with $x, y, z \in \mathbb{N}, 2 \nmid z$ and $y-z \neq \pm 1$. (For example, $4 \times 63+1=253=10^{2}+12^{2}+3^{2}$.) When $4 n+1$ is squarefree with $4 n+1 \notin S$, by Lemma 2.1 we have $R_{3}(4 n+1) \geqslant 12 h(-4 n-1)>12 \times 4=48$ and hence $4 n+1=x^{2}+y^{2}+z^{2}$ for some $x, y, z \in \mathbb{N}$ with $2 \nmid z$ and $y-z \neq \pm 1$.

In view of the above, we can write $4 n+1$ as $(2 x)^{2}+(2 y)^{2}+(2 z+1)^{2}$ with $x, y, z \in \mathbb{N}$ and $2 y-(2 z+1) \neq \pm 1$. It follows that $n=x^{2}+y^{2}+2 T_{z}=x^{2}+T_{y+z}+T_{z-y}$ with $z-y \notin\{0,-1\}$. Note that $T_{z-y} \neq 0$. If $y+z \leqslant 5$, then $T_{y+z}+T_{z-y}$ belongs to the set

$$
T:=\left\{T_{i}+T_{j}: i, j=0, \ldots, 5\right\}=\{0,1,3,4,6,7,9, \ldots, 13,15,16,18,20,21,25,30\} .
$$

If $n=x^{2}+t>66=6^{2}+30$ with $x \in \mathbb{N}$ and $t \in T$, then $x>6$ and hence by (c) we can write $x^{2}+t$ as $a^{2}+T_{b}+T_{c}$ with $a, b, c \in \mathbb{N}, b \geqslant c>0$ and $b \geqslant 6$.

Let $k \in\{1,2,3,4,5,6\}$. It is easy to verify that each $n=T_{k}+1, T_{k}+2, \ldots, 66$ can be written as $x^{2}+T_{y}+T_{z}$ with $x, y, z \in \mathbb{N}, y \geqslant k$ and $z \geqslant 1$. Thus, for any $n \in \mathbb{N}$ we can write $n+T_{k}+1$ as $x^{2}+T_{y+k}+T_{z+1}$ with $x, y, z \in \mathbb{N}$ and hence

$$
n=x^{2}+T_{y+k}-T_{k}+T_{z+1}-T_{1}=x^{2}+\frac{y(y+2 k+1)}{2}+\frac{z(z+3)}{2} .
$$

This proves the universality of $(2,0,1,2 k+1,1,3)$ over $\mathbb{N}$.

(e) As conjectured by Sun [S07] and proved in [OS], any positive integer can be written as the sum of a square, an odd square and a triangular number. So, for any $m \in \mathbb{Z}^{+}$there are $a, b, c \in \mathbb{N}$ with $a$ odd such that $T_{m}=a^{2}+b^{2}+T_{c}$ and hence $2 T_{m}=(a+b)^{2}+|a-b|^{2}+2 T_{c}$ with $a+b \geqslant a>0$. If $n \in \mathbb{Z}^{+}$is not twice a triangular number, then $4 n+1$ is not a square, hence by the Gauss-Legendre theorem there are $x, y, z \in \mathbb{N}$ with $\max \{x, y\}>0$ such that $4 n+1=(2 x)^{2}+(2 y)^{2}+(2 z+1)^{2}$ and thus $n=x^{2}+y^{2}+2 T_{z}$.

By the above, for any $n \in \mathbb{N}$ there are $x, y, z \in \mathbb{N}$ such that $n+1=(x+1)^{2}+y^{2}+2 T_{z}=$ $x(x+2)+1+T_{y+z}+T_{z-y}$. So $(2,4,2,2,2,0)$ and $(2,4,1,1,1,1)$ are universal over $\mathbb{N}$.

Clearly, $4 x^{2}+T_{y}+z(z+3) / 2$ is universal over $\mathbb{N}$ if and only if any positive integer can be written as $4 x^{2}+T_{y}+T_{z}$ with $x, y, z \in \mathbb{N}$ and $\max \{y, z\}>0$. By Sun [S07, Theorem $1(\mathrm{i})]$, we can write any $n \in \mathbb{Z}^{+}$in the form $(2 x)^{2}+T_{y}+T_{z}$ with $x, y, z \in \mathbb{N}$. 
If $y=z=0$, then $n=4 x^{2}=4 \times 0^{2}+T_{2 x-1}+T_{2 x}$. Thus $4 x^{2}+T_{y}+z(z+3) / 2$ (or the tuple $(8,0,1,3,1,1))$ is universal over $\mathbb{N}$.

(f) Let $n>3$ be an integer. If $4 n+1=m^{2}$ with $m \in\{4,5,6, \ldots\}$, then by Lemma 2.5 we can write $4 n+1=x^{2}+y^{2}+z^{2}$ with $x, y, z \in \mathbb{N}, 2 \nmid z$ and $\max \{x, y\}>0$, hence $\{x, y\} \nsubseteq\{0, \pm 2\}$ since $m^{2}-z^{2} \notin\left\{2^{2}, 2^{2}+2^{2}\right\}$. If $4 n+1$ is not a square but $d^{2} \mid 4 n+1$ for some integer $d>1$, then by Lemma 2.1 there are $x, y, z \in \mathbb{N}$ with $2 \nmid z$ such that $4 n+1=(d x)^{2}+(d y)^{2}+(d z)^{2}$ with $\max \{d x, d y\} \geqslant d>2$.

Now suppose that $4 n+1$ is squarefree. If $n=9$, then $4 n+1=0^{2}+6^{2}+1^{2}$. If $n \neq 9$, then by Lemmas 2.1 and 2.3 we have $R_{3}(4 n+1)=12 h(-4 n-1)>12 \times 2=24$ and hence the equation $x^{2}+y^{2}+z^{2}=4 n+1$ has integral solutions with $2 \nmid z$ and $\{x, y\} \nsubseteq\{0, \pm 2\}$. (As $4 n+1>16$, there is at most one value of $\delta \in\{0,1,2\}$ with $4 n+1-\delta 2^{2}$ a square.)

By the above, for any integer $n \geqslant 2$ there are $x, y, z \in \mathbb{N}$ such that $4(n+2)+1=$ $(2(x+2))^{2}+(2 y)^{2}+(2 z+1)^{2}$ and hence $n=x(x+4)+y^{2}+2 T_{z}=x(x+4)+T_{y+z}+T_{z-y}$. Note also that $n=0(0+4)+n^{2}+2 T_{0}=0(0+4)+T_{n}+T_{0}$ for each $n=0,1$. Therefore both $(2,8,2,2,2,0)$ and $(2,8,1,1,1,1)$ are universal tuples over $\mathbb{N}$.

Let $n \in \mathbb{N}$. As mentioned in the last paragraph, there are $x, y, z \in \mathbb{N}$ such that

$4(2 n+5)+1=(2 x+4)^{2}+(2 y)^{2}+(2 z+1)^{2}=2(x+y+2)^{2}+2(x-y+2)^{2}+(2 z+1)^{2}$.

Since $(x+y+2)^{2}+(x-y+2)^{2} \equiv 2(\bmod 4)$, there are $u, v \in \mathbb{N}$ such that $x+y+2=2 u+3$ and $|x-y+2|=2 v+1$. Thus $8 n+21=2(2 u+3)^{2}+2(2 v+1)^{2}+(2 z+1)^{2}$ and hence $n=u(u+3)+v(v+1)+T_{z}$. This proves the universality of $(2,6,2,2,1,1)$ over $\mathbb{N}$.

(g) Let $n \geqslant 2$ be an integer. By Lemma 2.5, there are $x, y, z \in \mathbb{N}$ with $x \geqslant y \geqslant z$ and $y>0$ such that $n=T_{x}+T_{y}+T_{z}$. If $\{x-z, y-z\} \subseteq\{1,3,5\}$, then $x-y \notin\{1,3,5\}$ since $x \equiv y(\bmod 2)$, and $n \leqslant T_{5}+T_{5}+T_{0}=30$ if $z=0$.

Let $n>31$ be an integer. By the last paragraph, $n=T_{x}+T_{y}+T_{z}$ for some $x, y \in \mathbb{Z}^{+}$and $z \in\{0, \ldots, y\}$ with $y-z \notin\{1,3,5\}$. If $y+z+1 \in\{1,3,5\}$, then $(y, z) \in\{(1,1),(2,0),(2,2),(3,1),(4,0)\}$ and hence $T_{y}+T_{z} \leqslant T_{4}=10$, thus $x>6$ (as $\left.n>T_{6}+10=31\right)$, and $x-z, x+z+1 \notin\{1,3,5\}$ unless $(y, z)=(2,2)$ and $x=7$ in which case $n=T_{7}+T_{2}+T_{2}=T_{7}+T_{3}+T_{0}$ with $7-0,7+0+1 \notin\{1,3,5\}$. Therefore, we can always write $n$ as $T_{u}+T_{v}+T_{w}$ with $u, v \in \mathbb{Z}^{+}, w \in\{0, \ldots, v\}$ and $v-w, v+w+1 \notin\{1,3,5\}$. It follows that

$$
T_{v}+T_{w}=\frac{(v+w+1)^{2}+(v-w)^{2}-1}{4}=\frac{(2 r)^{2}+(2 s+1)^{2}-1}{4}=r^{2}+s(s+1)
$$

for some $r, s \in \mathbb{N}$ with $s>2$. Hence $n=T_{u}+r^{2}+s(s+1)$. For each $k \in\{0,1,2,3\}$, clearly $t=s-k \in \mathbb{N}$ and

$$
n-k(k+1)=T_{u}+r^{2}+(t+k)(t+k+1)-k(k+1)=T_{u}+r^{2}+t(t+2 k+1) .
$$

Note also that $T_{u}=T_{a+1}=a(a+3) / 2+1$ with $a=u-1 \in \mathbb{N}$.

Let $k \in\{1,2,3\}$. By the above, any integer $n>30-k(k-1)$ can be written $a(a+3) / 2+r^{2}+t(t+2(k-1)+1)$ with $a, r, t \in \mathbb{N}$. We can easily see that each $n=0,1, \ldots, 30-k(k-1)$ also can be written as $x(x+3) / 2+y^{2}+z(z+2 k-1)$ 
with $x, y, z \in \mathbb{N}$. Thus the tuple $(2,4 k-2,2,0,1,3)$ is universal over $\mathbb{N}$. Similarly, $(2,4 k+2,2,0,1,1)$ is also a universal tuple over $\mathbb{N}$.

(h) Let $k \in\{1,2,3\}$. It is easy to verify that every $n=k^{2}, \ldots, 33$ can be written as $T_{x}+y^{2}+z^{2}$ with $x, y, z \in \mathbb{N}$ and $\max \{y, z\} \geqslant k$.

Now let $n \in \mathbb{N}$ with $n>33$. By [OS], $n=T_{x}+y^{2}+z^{2}$ for some $x, y, z \in \mathbb{N}$ with $y$ odd. Assume that $\max \{y, z\}<k$. Then $y=1$ and $z \leqslant k-1$, hence

$$
r:=n-T_{x} \in\left\{s^{2}+1: s=0, \ldots, k-1\right\} \subseteq\left\{s^{2}+1: s=0,1,2\right\}=\{1,2,5\} .
$$

As $n>33$, we have $T_{x}>28$ and hence $x>7$. Since $T_{x}<n=T_{x}+r \leqslant T_{x}+5<T_{x+1}$, $n$ is not a triangular number. By [S07, Theorem $1(\mathrm{ii})]$, there are $a, b, c, u, v, w \in \mathbb{N}$ with $a \not \equiv b(\bmod 2)$ and $u \equiv v(\bmod 2)$ such that $n=a^{2}+b^{2}+T_{c}=u^{2}+v^{2}+T_{w}$. Suppose that $\max \{a, b\}<k \leqslant 3$ and also $\max \{u, v\}<k \leqslant 3$. Then $a^{2}+b^{2} \in\left\{1^{2}+0^{2}, 1^{2}+2^{2}\right\}$ and $u^{2}+v^{2} \in\left\{0^{2}+0^{2}, 0^{2}+2^{2}, 1^{2}+1^{2}, 2^{2}+2^{2}\right\}$. Note that $T_{x+1}>n \geqslant T_{w}=n-\left(u^{2}+v^{2}\right) \geqslant$ $T_{x}+1-8>T_{x-1}$. So we have $w=x$ and hence $r=u^{2}+v^{2} \in\{0,2,4,8\}$. Similarly, $c=x$ and hence $r=a^{2}+b^{2} \in\{1,5\}$. Thus we get a contradiction since $\{0,2,4,8\} \cap\{1,5\}=\emptyset$.

In view of the above, $T_{x}+y^{2}+z(z+2 k)=T_{x}+y^{2}+(z+k)^{2}-k^{2}$ is universal over $\mathbb{N}$, i.e., the tuple $(2,4 k, 2,0,1,1)$ is universal over $\mathbb{N}$.

(i) Let $n \in \mathbb{N}$. By Lemma 2.1 we have $R_{3}(8(n+1)+3)>0$ and hence $n+1=$ $T_{x}+T_{y}+T_{z}$ for some $x, y, z \in \mathbb{N}$ with $y>z$. Clearly, $4\left(T_{y}+T_{z}\right)+1=(y+z+1)^{2}+(y-$ $z)^{2}=(2(u+1))^{2}+(2 v+1)^{2}$ for some $u, v \in \mathbb{N}$, and hence $n=T_{x}+u(u+2)+v(v+1)$. Therefore $(2,4,2,2,1,1)$ is universal over $\mathbb{N}$.

(j) Let $k \in\{1,2,3\}$. Clearly, $(2,4 k, 2,0,1,3)$ is universal over $\mathbb{N}$ if and only if any integer $n \geqslant k^{2}+1$ can be written as $x^{2}+y^{2}+T_{z}$ with $x, y, z \in \mathbb{N}, y \geqslant k$ and $z \geqslant 1$. By Lemma 2.8 and the equalities $T_{2}=1^{2}+1^{2}+T_{1}$ and $T_{3}=1^{2}+2^{2}+T_{1}$, any triangular number greater than $k^{2}$ can be written as $x^{2}+y^{2}+T_{z}$ with $x, y, z \in \mathbb{N}, y \geqslant k$ and $z \geqslant 1$.

Now we fix an integer $n \geqslant k^{2}+1$ which is not a triangular number. Then $8 n+1$ is not a square. By [S07, Theorem 1(ii)],

$$
r_{0}(n)=r_{1}(n)=\frac{1}{4}\left|\left\{(x, y, z) \in \mathbb{Z}^{3}: x^{2}+y^{2}+T_{z}=n\right\}\right|,
$$

where

$$
r_{\delta}(n):=\mid\left\{(x, y, z) \in \mathbb{Z} \times \mathbb{Z} \times \mathbb{N}: x^{2}+y^{2}+T_{z}=n \text { and } x-y \equiv \delta(\bmod 2)\right\} \mid
$$

for $\delta=0,1$. For $x, y, z \in \mathbb{Z}$, clearly

$$
n=x^{2}+y^{2}+T_{z} \Longleftrightarrow 8 n+1=(2 x+2 y)^{2}+(2 x-2 y)^{2}+(2 z+1)^{2} .
$$

If $8 n+1=u^{2}+v^{2}+w^{2}$ with $u, v, w \in \mathbb{N}$ and $2 \nmid w$, then $8 \mid u^{2}+v^{2}$, hence $2|u, 2| v$ and $u / 2 \equiv v / 2(\bmod 2)$. Thus, with the help of the Gauss-Legendre theorem we have

$$
12 r_{0}(n)=12 r_{1}(n)=\left|\left\{(x, y, z) \in \mathbb{Z}^{3}: x^{2}+y^{2}+z^{2}=8 n+1\right\}\right|>0 .
$$

If $n-0^{2}-1^{2}$ and $n-2^{2}-1^{2}$ are both triangular numbers, then $n=11$. If at least two of $n-1^{2}-1^{2}, n-0^{2}-2^{2}, n-2^{2}-2^{2}$ are triangular numbers, then $n \in\{14,23\}$. When 
$n \in\{11,14,23\}$, we can easily verify that $n=x^{2}+y^{2}+T_{z}$ for some $x, y, z \in \mathbb{N}$ with $y \geqslant k$ and $z \geqslant 1$. Let $n \neq 11,14,23$. If $r_{0}(n)=r_{1}(n)>4(k-1)$, or $r_{0}(n)=r_{1}(n)>4$ and $n-5 \notin\left\{T_{m}: m \in \mathbb{N}\right\}$, then $n=a^{2}+b^{2}+T_{c}=u^{2}+v^{2}+T_{w}$ for some $a, b, c, u, v, w \in \mathbb{N}$ with $a \not \equiv b(\bmod 2), u \equiv v(\bmod 2), \max \{a, b\} \geqslant k$ and $\max \{u, v\} \geqslant k$, hence $T_{c} \not \equiv T_{w}$ $(\bmod 2)$ and thus $n=x^{2}+y^{2}+T_{z}$ for some $x, y, z \in \mathbb{N}$ with $y \geqslant k$ and $z \geqslant 1$.

The above arguments with $k=1$ yield that any integer $n \geqslant 1^{2}+1$ can be written as $x^{2}+(y+1)^{2}+T_{z+1}$ with $x, y, z \in \mathbb{N}$. Thus $(2,0,2,0,1,3)$ and $(2,4,2,0,1,3)$ are universal tuples over $\mathbb{N}$.

Now we assume that $k \in\{2,3\}$. Write $8 n+1=d^{2} q$ with $d, q \in \mathbb{Z}^{+}$and $q$ squarefree. Then $q>1$ and $q \equiv 1(\bmod 8)$. By Lemma $2.3, h(-8 n-1)=h(-q) \geqslant 4$, and the equality holds only when $q$ belongs to the set $Q=\{17,33,57,73,97,177,193\}$. By Lemma 2.1, if $d>1$ then

$$
\left|\left\{(x, y, z) \in \mathbb{Z}^{3}: x^{2}+y^{2}+z^{2}=8 n+1\right\}\right| \geqslant R_{3}(8 n+1)+R_{3}(q) \geqslant 24 h(-q)
$$

and hence $r_{0}(n)=r_{1}(n) \geqslant 2 h(-q) \geqslant 8$. If $n=1^{2}+2^{2}+T_{m}$ for some $m \in \mathbb{N}$, then $(2 m+1)^{2}+40=8 n+1=d^{2} q$ and hence $q \notin Q$ since $\left(\frac{-40}{p}\right)=-1$ for all $p \in Q$ with $(\dot{\bar{p}})$ the Jacobi symbol, therefore $r_{0}(n)=r_{1}(n) \geqslant 2 h(-q)>8$ when $d>1$.

Now we handle the case $d=1$. If $8 n+1=q \in Q$, then $n \in\{2,4,7,9,12,22,24\}$. Recall that $n \geqslant k^{2}+1$. Clearly,

$7=0^{2}+2^{2}+T_{2}, 9=2^{2}+2^{2}+T_{1}, 12=0^{2}+3^{2}+T_{2}, 22=0^{2}+4^{2}+T_{3}, 24=0^{2}+3^{2}+T_{5}$.

Now assume that $8 n+1=q \notin Q$. Then $h(-q)>4$. If $h(-q) \leqslant 8$, then by Lemma $2.3(\mathrm{v}), n$ belongs to the set

$$
\{5,8,13,14,17,18,27,33,34,39,42,43,48,57,66,69,87,99\}
$$

and we can directly verify that $n=x^{2}+y^{2}+T_{z}$ for some $x, y, z \in \mathbb{N}$ with $y \geqslant k$ and $z \geqslant 1$. When $h(-q)>8$, by $(3.1)$ and Lemma 2.1 we have $r_{0}(n)=r_{1}(n)=R_{3}(8 n+1) / 12 \geqslant$ $h(-8 n-1)=h(-q)>8$. Thus $(2,4 k, 2,0,1,3)$ is indeed universal over $\mathbb{N}$.

(k) Let $k \in\{1,2,3\}$. We want to prove that $2 x^{2}+T_{y}+z(z+2 k+1) / 2=2 x^{2}+T_{y}+$ $T_{z+k}-T_{k}$ is universal over $\mathbb{N}$ (i.e., $(4,0,1,2 k+1,1,1)$ is a universal tuple over $\mathbb{N}$ ). It is easy to check that each $n=T_{k}, T_{k}+1, \ldots, 77$ can be written as $x^{2}+T_{y}+T_{z}$ with $x, y, z \in \mathbb{N}$ and $\max \{y, z\} \geqslant k$.

Now let $n \in \mathbb{Z}^{+}$with $n \geqslant 78$. If $8 n+2=x^{2}+a=y^{2}+b$ with $x, y \in \mathbb{N}, a<b$ and

$$
a, b \in A:=\left\{w^{2}+z^{2}: w, z \in\{1,3,5\}\right\}=\{2,10,18,26,34,50\},
$$

then $x>y \geqslant \sqrt{8 \times 78+2-50}=24$ and hence $x^{2}+a>y^{2}+2 y+a \geqslant y^{2}+48+a \geqslant y^{2}+b$. So $8 n+2$ can be written as $x^{2}+a$ with $a \in A$ in at most one way. Therefore, the equation $8 n+2=x^{2}+y^{2}+z^{2}$ has at most $3 ! \times 2^{3}=48$ integral solutions with the two odd numbers among $x, y, z$ in the set $\{ \pm 1, \pm 3, \pm 5\}$.

Write $8 n+2=d^{2} q$ with $d, q \in \mathbb{Z}^{+}$and $q$ squarefree. Then $8 \mid(q-2)$. By Lemma 2.3, $h(-q) \leqslant 4$ only when $q$ is among $2,10,34,42,82$, 130. Clearly, $82 d^{2}=0^{2}+d^{2}+(9 d)^{2}$ and $130 d^{2}=0^{2}+(7 d)^{2}+(9 d)^{2}$. If $h(-q)>4$, then by Lemma 2.1 we have

$$
\left|\left\{(x, y, z) \in \mathbb{Z}^{3}: x^{2}+y^{2}+z^{2}=8 n+2\right\}\right| \geqslant R_{3}(q)=12 h(-q)>48
$$


and hence $8 n+2=x^{2}+y^{2}+z^{2}$ for some $x, y, z \in \mathbb{N}$ with the two odd numbers of $x, y, z$ not all in $\{1,3,5\}$. As $R_{3}\left((8 n+2) / d^{2}\right)>0$ by Lemma 2.1 , there are $x, y, z \in \mathbb{N}$ with $\operatorname{gcd}(x, y, z)=1$ such that $8 n+2=(d x)^{2}+(d y)^{2}+(d z)^{2}$ with two of the odd numbers among $d x, d y, d z$ at least $d$. If $d \in\{1,3\}$, then $q=(8 n+2) / d^{2} \geqslant(8 \times 78+2) / 9=626 / 9>$ 69 . If $d=5$ then $q \geqslant 626 / 25>25$. Note that $5^{2} \times 34=0^{2}+15^{2}+25^{2}$ and $5^{2} \times 42=$ $20^{2}+5^{2}+25^{2}$. Therefore, we always can write $8 n+2=(2 w)^{2}+(2 y+1)^{2}+(2 z+1)^{2}$ with $w, y, z \in \mathbb{N}$ and $\max \{y, z\} \geqslant 3 \geqslant k$. Clearly, $w=2 x$ for some $x \in \mathbb{N}$, and hence $n=2 x^{2}+T_{y}+T_{z}$. We are done.

(1) To prove that $(3,9,2,0,1,3),(3,3,2,0,1,3)$ and $(3,9,2,0,1,1)$ are universal tuples over $\mathbb{N}$, we only need to show that any integer $n \geqslant 4$ can be written as $x^{2}+T_{y}+3 T_{z}$ with $x \in \mathbb{N}$ and $y, z \in \mathbb{Z}^{+}$which can be easily verified for all $n=4,5, \ldots, 45$.

Now, we fix an integer $n \geqslant 46$. By the Gauss-Legendre theorem, $12 n+6=x^{2}+y^{2}+z^{2}$ for some $x, y, z \in \mathbb{Z}$ with $2 \mid x$ and $2 \nmid y z$. Note that $12 n+6>12 \times 46+6>$ $9^{2}+(9+3)^{2}+(9+9)^{2}$. It is easy to see that the equation $12 n+6=x^{2}+y^{2}+z^{2}$ has at most $2 ! \times 2^{3}=16$ integral solutions with $x$ even, and $y, z \in\{ \pm(x-3), \pm(x+3)\}$ or

$$
\{y, z\} \in\left\{\left\{\varepsilon_{1}\left(x+3 \varepsilon_{0}\right), \varepsilon_{2}\left(x+9 \varepsilon_{0}\right)\right\}: \varepsilon_{0}, \varepsilon_{1}, \varepsilon_{2} \in\{ \pm 1\}\right\} .
$$

Write $12 n+6=d^{2} q$ with $d, q \in \mathbb{Z}^{+}$and $q$ squarefree. Obviously $q \equiv 2(\bmod 4)$. If $d>3$, then by Lemma 2.1 we have $12 n+6=x^{2}+y^{2}+z^{2}$ for some $x, y, z \in \mathbb{N}$ with $2 \mid x$ and $\operatorname{gcd}(x, y, z)=d>3$, hence $\{y, z\} \nsubseteq\{ \pm(x-3), \pm(x+3)\}$ and (3.2) fails. If $h(-q)>4$, then by Lemma 2.3 we have $R_{3}(12 n+6)=12 h(-12 n-6)=12 h(-q)>48$ and hence the equation $12 n+6=x^{2}+y^{2}+z^{2}$ has more than 16 solutions with $x$ even. If $h(q) \leqslant 4$, then by Lemma 2.3 the number $q$ belongs to the set

$$
E=\{2,6,10,14,22,30,34,42,46,58,70,78,82,102,130,142,190\}
$$

If $d=1$, then $q=12 n+6>12 \times 46+6>190$ and hence $h(-q)>4$. If $12 n+6=3^{2} q$ with $q \in E$, then we can verify that the equation $12 n+6=x^{2}+y^{2}+z^{2}$ has solutions with $x$ even, $\{y, z\} \nsubseteq\{ \pm(x-3), \pm(x+3)\}$ and (3.2) invalid.

By the above, there are $x, y, z \in \mathbb{Z}$ with $2 \mid x, 2 \nmid y z$ and $\{y, z\} \nsubseteq\{ \pm(x-3), \pm(x+3)\}$ such that (3.2) fails. As $x^{2}+y^{2}+z^{2} \equiv 0(\bmod 3)$, either $x \equiv y \equiv z \equiv 0(\bmod 3)$ or $3 \nmid x y z$. Without loss of generality, we may assume that $x \equiv y \equiv z(\bmod 3)$. Recall Jacobi's identity

$$
3\left(x^{2}+y^{2}+z^{2}\right)=(x+y+z)^{2}+2\left(\frac{x+y}{2}-z\right)^{2}+6\left(\frac{x-y}{2}\right)^{2}
$$

which can be verified directly. Clearly, $x+y+z=6 w$ for some $w \in \mathbb{Z}$, and $x+y-2 z=$ $6 u+3$ and $x-y=6 v+3$ for some $u, v \in \mathbb{Z}$. Thus, by (3.3) we have

$$
36 n+18=3\left(x^{2}+y^{2}+z^{2}\right)=(6 w)^{2}+2\left(\frac{6 u+3}{2}\right)^{2}+6\left(\frac{6 v+3}{2}\right)^{2}
$$

and hence $n=w^{2}+T_{u}+3 T_{v}$. If $x-y \neq \pm 3$, then $v \neq 0,-1$ and hence $T_{v} \neq 0$. If $x+y-2 z \neq \pm 3$, then $u \neq 0,-1$ and hence $T_{u} \neq 0$. So we are done if $\{x-$ 
$y, x+y-2 z\} \cap\{ \pm 3\}=\emptyset$. Due to the symmetry of $y$ and $z$, we are also done if $\{x-z, x+z-2 y\} \cap\{ \pm 3\}=\emptyset$.

If $\{x+y-2 z, x+z-2 y\} \subseteq\{ \pm 3\}$, then $3(y-z)=x+y-2 z-(x+z-2 y) \in\{0, \pm 6\}$, hence $y=z($ since $y \equiv z(\bmod 3))$ and $x-y=x-z \in\{ \pm 3\}$, which contradicts that $\{y, z\} \nsubseteq\{ \pm(x-3), \pm(x+3)\}$. If $x-y, x-z \neq \pm 3$, then we are done since $x+y-2 z \neq \pm 3$ or $x+z-2 y \neq \pm 3$.

Now we consider the remaining case in which exactly one of $|x-y|$ and $|x-z|$ is 3 . Without loss of generality, we assume that $x-y \neq \pm 3$ and $x-z \in\{ \pm 3\}$. Thus $y \neq z$. We are done if $x+y-2 z \neq \pm 3$. Suppose that $x+y-2 z=(x-z)+(y-z) \in\{ \pm 3\}$. Then $y-z=-2(x-z) \in\{ \pm 6\}$ and $x-y=x-z-(y-z)=3(x-z) \in\{ \pm 9\}$. So $(y, z)=(x+9, x+3)$ or $(x-9, x-3)$, which contradicts that (3.2) fails.

In view of the above, we have completed the proof of Theorem 1.1.

\section{Proofs of Theorems 1.4 And 1.7}

Lemma 4.1. Let $a, b, c, d \in \mathbb{Z}$ with $a \geqslant c \geqslant 1, b>-a, d>-c, a \equiv b(\bmod 2)$ and $c \equiv d(\bmod 2)$. Then $\{1, \ldots, 18\} \nsubseteq\left\{\left\{\psi_{a, b}(x)+\psi_{c, d}(y): x, y \in \mathbb{N}\right\}\right.$.

Proof. It is easy to see that neither $\{x(a x+b) / 2: x \in \mathbb{N}\}$ nor $\{y(c y+d) / 2: y \in \mathbb{N}\}$ contains $\{1,2\}$. So $\{1,2\} \nsubseteq\left\{\psi_{a, b}(x)+\psi_{c, d}(y): x, y \in \mathbb{N}\right\}$ if $\psi_{a, b}(1)=(a+b) / 2>2$ or $\psi_{c, d}(1)=(c+d) / 2>2$.

Below we suppose that $a+b \leqslant 4$ and $c+d \leqslant 4$. In the case $a c<212$, via a computer we find that one of $1, \ldots, 9$ cannot be written as $\psi_{a, b}(x)+\psi_{c, d}(y)$ with $x, y \in \mathbb{N}$.

Now we assume that $a c \geqslant 212$. Then $1 / a+1 / c \leqslant 213 / 212$.

Fix a positive integer $N$. For $x \in \mathbb{Z}$, it is easy to see that

$$
\frac{x(a x+b)}{2} \leqslant N \Longleftrightarrow-\frac{\sqrt{8 a N+b^{2}}+b}{2 a} \leqslant x \leqslant \frac{\sqrt{8 a N+b^{2}}-b}{2 a} .
$$

As $-a<b \leqslant a$ or $b>0$, we have

$$
\left|\left\{x \in \mathbb{N}: \psi_{a, b}(x) \leqslant N\right\}\right| \leqslant 1+\frac{\sqrt{8 a N+b^{2}}-b}{2 a}<\frac{3}{2}+\sqrt{\frac{2 N}{a}+\frac{1}{4}} ;
$$

Similarly, $\left|\left\{x \in \mathbb{N}: \psi_{c, d}(x) \leqslant N\right\}\right|<3 / 2+\sqrt{2 N / c+1 / 4}$. Note that

$$
\sqrt{u}+\sqrt{v} \leqslant \sqrt{2 u+2 v} \text { for all } u, v \geqslant 0 .
$$

Therefore

$$
\begin{aligned}
& \left|\left\{\psi_{a, b}(x)+\psi_{c, d}(y): x, y \in \mathbb{N}\right\} \cap[0, N]\right| \\
< & \left(\frac{3}{2}+\sqrt{\left.\frac{2 N}{a}+\frac{1}{4}\right)\left(\frac{3}{2}+\sqrt{\frac{2 N}{c}+\frac{1}{4}}\right)}\right. \\
\leqslant & \frac{9}{4}+\sqrt{\frac{4 N^{2}}{a c}+\frac{N}{2}\left(\frac{1}{a}+\frac{1}{c}\right)+\frac{1}{16}+\frac{3}{2}} \sqrt{4 N\left(\frac{1}{a}+\frac{1}{c}\right)+1} \\
\leqslant & f(N):=\frac{9}{4}+\sqrt{\frac{4 N^{2}}{212}+\frac{N}{2} \cdot \frac{213}{212}+\frac{1}{16}}+\frac{3}{2} \sqrt{4 N \times \frac{213}{212}+1} .
\end{aligned}
$$


Now, take $N=18$. Then

$$
\left|\left\{\psi_{a, b}(x)+\psi_{c, d}(y): x, y \in \mathbb{N}\right\} \cap[0, N]\right| \leqslant f(N)<1+N
$$

So one of $1, \ldots, N$ cannot be written as $\psi_{a, b}(x)+\psi_{c, d}(y)$ with $x, y \in \mathbb{N}$.

Proof of Theorem 1.4. In view of Lemma 4.1, for certain $m \in\{1, \ldots, 18\}$ we can write $m=\psi_{a, b}(x)+\psi_{c, d}(y)+\psi_{e, f}(z)$ with $x, y, z \in \mathbb{N}$ and $x>0$, and hence $(a+b) / 2 \leqslant$ $\psi_{a, b}(x) \leqslant m \leqslant 18$. Similarly, $(c+d) / 2 \leqslant 18$ and $(e+f) / 2 \leqslant 18$. Therefore

$$
b \leqslant 36-a, d \leqslant 36-c \text { and } f \leqslant 36-e .
$$

If $c e \geqslant 2000$ and $N=64$, then $1 / c+1 / e \leqslant 2001 / 2000$ and hence

$$
\begin{aligned}
&\left|\left\{\psi_{c, d}(y)+\psi_{e, f}(z): y, z \in \mathbb{Z}\right\} \cap[0, N]\right| \\
&<\frac{9}{4}+\sqrt{\frac{4 N^{2}}{2000}+\frac{N}{2} \cdot \frac{2001}{2000}+\frac{1}{16}}+\frac{3}{2} \sqrt{4 N \times \frac{2001}{2000}+1} \leqslant 33
\end{aligned}
$$

by the proof of Lemma 4.1, thus

$$
\left|\left\{\psi_{a, b}(x)+\psi_{c, d}(y)+\psi_{e, f}(z): x \in\{0,1\} \& y, z \in \mathbb{Z}\right\} \cap[0, N]\right|
$$

is at most $2 \times 32=N$. So, in the case $c e \geqslant 2000$, we can write certain $n \in\{0, \ldots, 64\}$ as $\psi_{a, b}(x)+\psi_{c, d}(y)+\psi_{e, f}(z)$ with $x, y, z \in \mathbb{N}$ and $x \geqslant 2$, hence $a+2 \leqslant 2 a+b \leqslant \psi_{a, b}(x) \leqslant$ $n \leqslant 64$ and thus $e \leqslant c \leqslant a \leqslant 62$.

Now we consider the case $c e<2000$. In view of (4.3), via a computer we find that for each $i=1, \ldots, 18$ there is an integer $n_{i} \in[i, 58]$ such that

$$
\left\{n_{i}, n_{i}-i\right\} \cap\left\{\psi_{c, d}(y)+\psi_{e, f}(z): y, z \in \mathbb{N}\right\}=\emptyset \text {. }
$$

(We note that $\{58,58-17\} \cap\left\{y^{2}+p_{3}(z): y, z \in \mathbb{N}\right\}=\emptyset$.) For $i=(a+b) / 2 \leqslant 18$, we can write $n_{i}$ as $\psi_{a, b}(x)+\psi_{c, d}(y)+\psi_{e, f}(z)$ with $x, y, z \in \mathbb{N}$ and $x>1$, thus $a+2 \leqslant$ $2 a+b \leqslant \psi_{a, b}(x) \leqslant n_{i} \leqslant 58$ and hence $e \leqslant c \leqslant a \leqslant 56$.

By the above, either $c e \geqslant 2000$ and $e \leqslant c \leqslant a \leqslant 62$, or $c e<2000$ and $e \leqslant c \leqslant a \leqslant 58$. In view of this and (4.3), via a computer we find that if every $n=0, \ldots, 10^{5}$ can be written as $\psi_{a, b}(x)+\psi_{c, d}(y)+\psi_{e, f}(z)$ with $x, y, z \in \mathbb{Z}$ then the tuple $(a, b, c, d, e, f)$ must be among the 56+10 tuples listed in Theorem 1.1 and Conjecture 1.2 if $a|b, c| d$ and $e \mid f$, or among the 407 tuples listed the Appendix if $a \nmid b$ or $c \nmid d$ or $e \nmid f$. This concludes the proof of Theorem 1.4.

Lemma 4.2. Let $a, b, c, d \in \mathbb{N}$ with $a \geqslant c \geqslant 2, a>b, c>d, a \equiv b(\bmod 2)$ and $c \equiv d$ $(\bmod 2)$. Then one of $1, \ldots, 28$ cannot be written as $\psi_{a, b}(x)+\psi_{c, d}(y)$ with $x, y \in \mathbb{Z}$.

Proof. If $a c<1000$, then $\max \{a, b, c, d\}=a<500$, and hence we may use a computer to get that one of $1, \ldots, 21$ cannot be written as $\psi_{a, b}(x)+\psi_{c, d}(y)$ with $x, y \in \mathbb{Z}$. Note that $21 \neq x(7 x+1) / 2+y(3 y+1) / 2$ for all $x, y \in \mathbb{Z}$.

Below we assume that $a c \geqslant 1000$. It is easy to see that $1 / a+1 / c \leqslant 251 / 500$. 
Let $N$ be any positive integer. In view of (4.1),

$$
\left|\left\{x \in \mathbb{Z}: \psi_{a, b}(x) \leqslant N\right\}\right|-1 \leqslant \frac{\sqrt{8 a N+b^{2}}-b}{2 a}-\left(-\frac{\sqrt{8 a N+b^{2}}+b}{2 a}\right)<\sqrt{\frac{8 N}{a}+1} .
$$

Similarly, $\left|\left\{y \in \mathbb{Z}: \psi_{c, d}(y) \leqslant N\right\}\right|<1+\sqrt{8 N / c+1}$. With the help of (4.2), we have

$$
\begin{aligned}
& \left|\left\{\psi_{a, b}(x)+\psi_{c, d}(y): x, y \in \mathbb{Z}\right\} \cap[0, N]\right| \\
< & \left(1+\sqrt{\frac{8 N}{a}+1}\right)\left(1+\sqrt{\frac{8 N}{c}+1}\right) \\
\leqslant & 1+\sqrt{\frac{64 N^{2}}{a c}+8 N\left(\frac{1}{a}+\frac{1}{c}\right)+1}+\sqrt{16 N\left(\frac{1}{a}+\frac{1}{c}\right)+4} \\
\leqslant & g(N):=1+\sqrt{\frac{64 N^{2}}{1000}+8 N \frac{251}{500}+1}+\sqrt{16 N \frac{251}{500}+4 .}
\end{aligned}
$$

Now, take $N=28$. Then

$$
\left|\left\{\psi_{a, b}(x)+\psi_{c, d}(y): x, y \in \mathbb{Z}\right\} \cap[0, N]\right|<g(N) \leqslant 1+N .
$$

Therefore, one of $1, \ldots, N$ cannot be written as $\psi_{a, b}(x)+\psi_{c, d}(y)$ with $x, y \in \mathbb{Z}$.

Proof of Theorem 1.7. For any integer $x$ with $|x| \geqslant 2$, we have

$$
\psi_{a, b}(x) \geqslant \frac{|x|(a|x|-b)}{2} \geqslant a|x|-b \geqslant 2 a-b>\psi_{a, b}(1)=\frac{a+b}{2} .
$$

In view of Lemma 4.2 , for certain $m \in\{1, \ldots, 28\}$ we can write $m=\psi_{a, b}(x)+$ $\psi_{c, d}(y)+\psi_{e, f}(z)$ with $x, y, z \in \mathbb{Z}$ and $x \neq 0$, and hence $(a-b) / 2=\psi_{a, b}(-1) \leqslant$ $\psi_{a, b}(x) \leqslant m \leqslant 28$. Similarly, $(c-d) / 2 \leqslant 28$ and $(e-f) / 2 \leqslant 28$.

If $c e \geqslant 1000$ and $N=190$, then

$$
\left|\left\{\psi_{c, d}(y)+\psi_{e, f}(z): y, z \in \mathbb{Z}\right\} \cap[0, N]\right|<g(N) \leqslant 96
$$

by the proof of Lemma 4.2 , and hence

$$
\left|\left\{\psi_{a, b}(x)+\psi_{c, d}(y)+\psi_{e, f}(z): x \in\{0,-1\} \& y, z \in \mathbb{Z}\right\} \cap[0, N]\right|
$$

is at most $2 \times 95=N$. So, in the case $c e \geqslant 1000$, we can write certain $n \in\{0,1, \ldots, 190\}$ as $\psi_{a, b}(x)+\psi_{c, d}(y)+\psi_{e, f}(z)$ with $x, y, z \in \mathbb{Z}$ and $x \neq 0,-1$, hence $(a+b) / 2 \leqslant \psi_{a, b}(x) \leqslant$ $n \leqslant 190$ and thus $a=(a-b) / 2+(a+b) / 2 \leqslant 28+190=218$.

Now we consider the case $c e<1000$. Via a computer we find that for each $i=$ $1, \ldots, 28$ there is an integer $n_{i} \in[i, 157]$ such that

$$
\left\{n_{i}, n_{i}-i\right\} \cap\left\{\psi_{c, d}(y)+\psi_{e, f}(z): y, z \in \mathbb{Z}\right\}=\emptyset .
$$

For $i=(a-b) / 2 \leqslant 28$, we can write $n_{i}$ as $\psi_{a, b}(x)+\psi_{c, d}(y)+\psi_{e, f}(z)$ with $x, y, z \in \mathbb{Z}$ and $x \neq 0,-1$, thus $(a+b) / 2 \leqslant \psi_{a, b}(x) \leqslant n_{i} \leqslant 157$ and hence $a=(a-b) / 2+(a+b) / 2 \leqslant$ $28+157=185$.

By the above, either $c e \geqslant 1000$ and $e \leqslant c \leqslant a \leqslant 218$, or $c e<1000$ and $e \leqslant$ $c \leqslant a \leqslant 185$. Via a computer we find that if each $n=0, \ldots, 10^{5}$ can be written as $\psi_{a, b}(x)+\psi_{c, d}(y)+\psi_{e, f}(z)$ with $x, y, z \in \mathbb{Z}$ then the tuple $(a, b, c, d, e, f)$ must be among the 12082 tuples listed in [S17a]. This completes the proof of Theorem 1.7. 


\section{Proofs of Theorems 1.9 And 1.11}

Proof of Theorem 1.9. Let $n$ be any nonnegative integer.

(i) $n=x(a x+b)+y(a y+a-b)$ if and only if $4 a n+b^{2}+(a-b)^{2}$ coincides with

$$
(a(2 x)+b)^{2}+(a(2 y)+a-b)^{2}=(a(-2 y-1)+b)^{2}+(a(-2 x-1)+a-b)^{2} .
$$

Therefore

$$
\begin{aligned}
& n \in\{x(a x+b)+y(a y+a-b): x, y \in \mathbb{Z}\} \\
\Longleftrightarrow & 4 a n+(a-b)^{2}+b^{2} \in\left\{(a u+b)^{2}+(a v+a-b)^{2}: u, v \in \mathbb{Z} \& 2 \mid u-v\right\} \\
\Longleftrightarrow & 4 a n+(a-b)^{2}+b^{2} \in\left\{(a(x-y)+b)^{2}+(a(x+y)+a-b)^{2}: x, y \in \mathbb{Z}\right\} \\
\Longleftrightarrow & n \in\left\{a T_{x}+\psi_{a, a-2 b}(y): x, y \in \mathbb{Z}\right\} .
\end{aligned}
$$

This proves (1.6).

(ii) Obverse that $2\left(x^{2}+4 T_{y}\right)+1=2 x^{2}+(2 y+1)^{2}$ and

$$
\begin{aligned}
& 2 n+1 \in\left\{u^{2}+2 v^{2}: u, v \in \mathbb{Z}\right\} \\
& \Longleftrightarrow 2 n+1 \in\left\{\left(\frac{x-y}{3}+y\right)^{2}+2\left(\frac{x-y}{3}\right)^{2}: x, y \in \mathbb{Z} \& 3 \mid x-y\right\} \\
& \Longleftrightarrow 6 n+3 \in\left\{x^{2}+2 y^{2}: x, y \in \mathbb{Z} \& 3 \mid x-y\right\} \\
& \Longleftrightarrow 6 n+3 \in\left\{x^{2}+2 y^{2}: x, y \in \mathbb{Z}\right\} . \\
& \Longleftrightarrow 6 n+3 \in\left\{x^{2}+2 y^{2}: x, y \in \mathbb{Z}, 2 \nmid x \& 3 \nmid x y\right\} \\
& \quad(\text { by }[\mathrm{JP}, \text { p. } 173] \text { or }[\mathrm{S} 15, \text { Lemma } 2.1]) \\
& \Longleftrightarrow 6 n+3 \in\left\{(6 x-1)^{2}+2(3 y-1)^{2}: x, y \in \mathbb{Z}\right\} \\
& \Longleftrightarrow n=4 p_{5}(x)+p_{8}(y) \text { for some } x, y \in \mathbb{Z} .
\end{aligned}
$$

So (1.9) holds. Similarly,

$$
\begin{aligned}
n \in\left\{x^{2}+T_{y}: x, y \in \mathbb{Z}\right\} & \Longleftrightarrow 8 n+1 \in\left\{u^{2}+2 v^{2}: u, v \in \mathbb{Z}\right\} \\
& \Longleftrightarrow 24 n+3 \in\left\{u^{2}+2 v^{2}: u, v \in \mathbb{Z}, 2 \nmid u v \& 3 \nmid u v\right\} \\
& \Longleftrightarrow 24 n+3 \in\left\{(6 x-1)^{2}+2(6 y-1)^{2}: x, y \in \mathbb{Z}\right\} \\
& \Longleftrightarrow n \in\left\{p_{5}(x)+2 p_{5}(y): x, y \in \mathbb{Z}\right\},
\end{aligned}
$$

and

$$
\begin{aligned}
n \in\left\{T_{x}+2 T_{y}: x, y \in \mathbb{Z}\right\} & \Longleftrightarrow 8 n+3 \in\left\{u^{2}+2 v^{2}: u, v \in \mathbb{Z}\right\} \\
& \Longleftrightarrow 24 n+9 \in\left\{x^{2}+2 y^{2}: x, y \in \mathbb{Z} \& 3 \nmid x y\right\} \\
& \Longleftrightarrow 24 n+9 \in\left\{x^{2}+8 y^{2}: x, y \in \mathbb{Z}, 2 \nmid x \& 3 \nmid x y\right\} \\
& \Longleftrightarrow 24 n+9 \in\left\{(6 x-1)^{2}+8(3 y-1)^{2}: x, y \in \mathbb{Z}\right\} \\
& \Longleftrightarrow n=p_{5}(x)+p_{8}(y) \text { for some } x, y \in \mathbb{Z} .
\end{aligned}
$$


This proves (1.7) and (1.8).

Now we show (1.10). Clearly, $8\left(T_{x}+T_{y}\right)+2=(2 x+1)^{2}+(2 y+1)^{2}$ and $40\left(\psi_{5,1}(x)+\psi_{5,3}(y)\right)+10=(10 x+1)^{2}+(10 y+3)^{2}=5\left((4 x+2 y+1)^{2}+(4 y-2 x+1)^{2}\right)$.

If $8 n+2=u^{2}+v^{2}$ with $u$ and $v$ odd, then $40 n+10=(2 u+v)^{2}+(u-2 v)^{2}=s^{2}+t^{2}$ for some $s, t \in \mathbb{Z}$ with $5 \nmid s t$ (by [S17, Lemma 2.1]), and hence $40 n+10=(10 x+1)^{2}+(10 y+3)^{2}$ for some $x, y \in \mathbb{Z}$. Therefore (1.10) holds.

The proof of Theorem 1.9 is now complete.

Lemma 5.1. Let $w=3 u^{2}+5 v^{2} \in \mathbb{Z}^{+}$with $u, v \in \mathbb{Z}$ and $8 \mid w$. Then $w=3 x^{2}+5 y^{2}$ for some odd integers $x$ and $y$.

Proof. Let $k=\operatorname{ord}_{2} \operatorname{gcd}(u, v)$ and write $u=2^{k} u_{0}$ and $v=2^{k} v_{0}$ with $u_{0}, v_{0} \in \mathbb{Z}$ not all even. If $k \in\{0,1\}$, then $u_{0}$ and $v_{0}$ are both odd since $8 \mid w$. If $u_{0} \not \equiv v_{0}(\bmod 2)$, then $k \geqslant 2$ and $4^{2}\left(3 u_{0}^{2}+5 v_{0}^{2}\right)=3 u_{2}^{2}+5 v_{2}^{2}$ with $u_{2}=u_{0}-5 v_{0}$ and $v_{2}=3 u_{0}+v_{0}$ both odd.

Let $j \in \mathbb{N}$. If $4^{j}\left(3 u_{0}^{2}+5 v_{0}^{2}\right)$ can be written as $3 u_{j}^{2}+5 v_{j}^{2}$ with $u_{j}$ and $v_{j}$ odd, then we may assume $u_{j} \not \equiv v_{j}(\bmod 4)$ without loss of generality, hence

$$
4^{j+1}\left(3 u_{0}^{2}+5 v_{0}^{2}\right)=4\left(3 u_{j}^{2}+5 v_{j}^{2}\right)=3 u_{j+1}^{2}+5 v_{j+1}^{2}
$$

with $u_{j+1}=\left(v_{j}-u_{j}\right) / 2+2 v_{j}$ and $v_{j+1}=\left(v_{j}-u_{j}\right) / 2+2 u_{j}$ both odd.

By the above, $w=4^{k}\left(3 u_{0}^{2}+5 v_{0}^{2}\right)=3 u_{k}^{2}+5 v_{k}^{2}$ for some odd integers $u_{k}$ and $v_{k}$.

Remark 5.2. Note also the following useful fact:

$$
3\left(\frac{x}{2}+y\right)^{2}+5\left(\frac{x}{2}-y\right)^{2}=3\left(\frac{x-3 y}{2}\right)^{2}+5\left(\frac{x+y}{2}\right)^{2}=2 x^{2}-2 x y+8 y^{2} .
$$

The following lemma is a well known result in the theory of quadratic forms.

Lemma 5.3. ([C, Theorem 1.3]) Let $f$ be an integral quadratic form with nonzero discriminant. If an integer $m$ is represented by $f$ over the field of real numbers as well as the ring $\mathbb{Z}_{p}$ of $p$-adic integers for each prime $p$, then $m$ is represented over $\mathbb{Z}$ by some form $f^{*}$ in the same genus as $f$.

Lemma 5.4. Let $n \in \mathbb{N}$ and $\delta \in\{0,1\}$. Then $12 n+8+3 \delta \in\left\{3 x^{2}+3 y^{2}+5 z^{2}: x, y, z \in\right.$ $\mathbb{Z}\}$.

Proof. There are two classes in the genus of $3 x^{2}+3 y^{2}+5 z^{2}$, the one not containing $3 x^{2}+3 y^{2}+5 z^{2}$ has the representative $3 x^{2}+2 y^{2}+8 z^{2}-2 y z$. If $12 n+8+3 \delta=3 x^{2}+$ $2 y^{2}+8 z^{2}-2 y z$ with $x, y, z \in \mathbb{Z}, 2 \nmid y$ and $y \not \equiv z(\bmod 2)$, then $3 \delta \equiv 3 x^{2}+2 y^{2} \equiv-x^{2}+2$ $(\bmod 4)$ which is impossible. Combining this with (5.1) and Lemma 5.3, we immediately obtain the desired result.

Proof of Theorem 1.11. Fix a nonnegative integer $n$.

(i) It is easy to see that

$$
\begin{gathered}
n=\frac{x(x+1)}{2}+\frac{y(3 y+1)}{2}+\frac{z(5 z+1)}{2} \\
\Longleftrightarrow 120 n+23=15(2 x+1)^{2}+5(6 y+1)^{2}+3(10 z+1)^{2} .
\end{gathered}
$$


There are two classes in the genus of $3 x^{2}+5 y^{2}+15 z^{2}$, and the one not containing $3 x^{2}+5 y^{2}+15 z^{2}$ has the representative $2 x^{2}-2 x y+8 y^{2}+15 z^{2}$. If $120 n+23=2 x^{2}+8 y^{2}+$ $15 z^{2}-2 x y$ for some $x, y \in \mathbb{Z}$ with $2 \nmid x$ and $y \not \equiv x(\bmod 2)$, then $23 \equiv 2 x^{2}+15 z^{2} \equiv 17$ $(\bmod 4)$ which is impossible. Thus, in view of (5.1) and Lemma 5.3, there are $x, y, z \in \mathbb{Z}$ such that $120 n+23=3 x^{2}+5 y^{2}+15 z^{2}$.

If $2 \nmid x$, then $5\left(y^{2}+3 z^{2}\right) \equiv 23-3 x^{2} \equiv 20(\bmod 8)$ and hence $y^{2}+3 z^{2}=s^{2}+3 t^{2}$ for some odd integers $s$ and $t$ (cf. [S15, Lemma 3.2]). If $2 \nmid z$, then $3 x^{2}+5 y^{2} \equiv 23-15 z^{2} \equiv 0$ $(\bmod 8)$ and hence $3 x^{2}+5 y^{2}=3 u^{2}+5 v^{2}$ for some odd integers $u$ and $v$ (by Lemma 5.1). If $x$ and $z$ are both even, then $y^{2} \equiv 5 y^{2} \equiv 23 \equiv 3(\bmod 4)$ which is impossible. So we may simply assume $2 \nmid x y z$ without loss of generality.

Since $3 x^{2} \equiv 23 \equiv 3(\bmod 5), x$ or $-x$ is congruent to 1 modulo 10 . As $y \not \equiv 0$ $(\bmod 3), y$ or $-y$ is congruent to 1 modulo 6 . Thus, for some $u, v, w \in \mathbb{Z}$ we have

$$
120 n+23=3(10 w+1)^{2}+5(6 v+1)^{2}+15(2 u+1)^{2}
$$

and hence $n=u(u+1) / 2+v(3 v+1) / 2+w(5 w+1) / 2$. This ends our proof of (1.12).

(ii) Let $\delta \in\{0,1\}$ and $r \in\{1,3,5\}$. There are two classes in the genus of $3 x^{2}+5 y^{2}+$ $30 z^{2}$, and the one not containing $3 x^{2}+5 y^{2}+30 z^{2}$ is

$$
2 x^{2}+15 y^{2}+15 z^{2}=2 x^{2}+30\left(\frac{y+z}{2}\right)^{2}+30\left(\frac{y-z}{2}\right)^{2} \text {. }
$$

When $120 n+30 \delta+3 r^{2}+5=2 x^{2}+30 u^{2}+30 v^{2}$ with $x, u, v \in \mathbb{Z}$, if $u \equiv v(\bmod 2)$ then $x \equiv u \equiv v \equiv \delta(\bmod 2)\left(\right.$ since $2 \mid x-\delta$ and $\left.u^{2}+v^{2} \equiv 2 \delta(\bmod 4)\right)$, thus we may assume $x \equiv u(\bmod 2)$ without loss of generality, and hence $2 x^{2}+30 u^{2}=3 a^{2}+5 b^{2}$ with $a=(x+5 u) / 2$ and $b=(x-3 u) / 2$ both integral. So, with the help of Lemma 5.3, there are $x, y, z \in \mathbb{Z}$ such that $120 n+30 \delta+3 r^{2}+5=3 x^{2}+5 y^{2}+30 z^{2}$.

Clearly, $z=2 w+\delta$ for some $w \in \mathbb{Z}$. Since $3 x^{2}+5 y^{2} \equiv 0(\bmod 8)$ and $3 x^{2}+5 y^{2} \neq 0$, by Lemma 5.1 we can write $3 x^{2}+5 y^{2}=3 s^{2}+5 t^{2}$ with $s$ and $t$ odd. Now, $120 n+30 \delta+$ $3 r^{2}+5=3 s^{2}+5 t^{2}+120 w(w+\delta)+30 \delta$. As $3 s^{2} \equiv 3 r^{2}(\bmod 5), s$ or $-s$ is congruent to $r$ modulo 10. Also, $t$ or $-t$ is congruent to 1 modulo 6 . So there are $u, v \in \mathbb{Z}$ such that

$$
120 n+3 r^{2}+5=3(10 v+r)^{2}+5(6 u+1)^{2}+120 w(w+\delta)
$$

and hence $n=w(w+\delta)+u(3 u+1) / 2+v(5 v+r) / 2$. This proves (1.13).

(iii) Let $r, s, t \in\{1,3\}$ with $\{r, s\} \neq\{3\}$. There are two classes in the genus of $3 x^{2}+5 y^{2}+5 z^{2}$, and the one not containing $3 x^{2}+5 y^{2}+5 z^{2}$ has the representative $2 x^{2}-2 x y+8 y^{2}+5 z^{2}$. If $120 n+5 r^{2}+5 s^{2}+3 t^{2}=2 x^{2}+8 y^{2}-2 x y+5 z^{2}$ with $x, y, z \in \mathbb{Z}, 2 \nmid x$ and $2 \mid y$, then $13 \equiv 5 r^{2}+5 s^{2}+3 t^{2} \equiv 2 x^{2}+5 z^{2} \equiv 2+5(\bmod 4)$ which is impossible. So, in light of (5.1) and Lemma 5.3, there are $x, y, z \in \mathbb{Z}$ such that $120 n+5 r^{2}+5 s^{2}+3 t^{2}=3 x^{2}+5 y^{2}+5 z^{2}$. As $3 x^{2} \not \equiv 13 \equiv 5 r^{2}+5 s^{2}+3 t^{2}(\bmod 4)$, $y$ and $z$ cannot be both even. Without loss of generality, we assume that $2 \nmid z$. Then $3 x^{2}+5 y^{2}>0$ and $3 x^{2}+5 y^{2} \equiv 0(\bmod 8)$. By Lemma 5.1 , we can write $3 x^{2}+5 y^{2}$ as $3 x_{0}^{2}+5 y_{0}^{2}$ with $x_{0}$ and $y_{0}$ both odd. 
By the last paragraph, $120 n+5 r^{2}+5 s^{2}+3 t^{2}=3 x^{2}+5 y^{2}+5 z^{2}$ for some odd integers $x, y, z$. Clearly $x$ or $-x$ has the form $10 w+t$ with $w \in \mathbb{Z}$. Since $y^{2}+z^{2} \equiv r^{2}+s^{2}$ $(\bmod 3)$, we have $y^{2}+z^{2}=(6 u+r)^{2}+(6 v+s)^{2}$ for some $u, v \in \mathbb{Z}$. Therefore

$$
120 n+5 r^{2}+5 s^{2}+3 t^{2}=3(10 w+t)^{2}+5(6 u+r)^{2}+5(6 v+s)^{2}
$$

and hence $n=u(3 u+r) / 2+v(3 v+s) / 2+w(5 w+t) / 2$. This proves (1.14).

(iv) As $3\left(s^{2}+t^{2}\right)+5 \equiv 3 \times 2+5=11(\bmod 12)$, by Lemma 5.4 there are $x, y, z \in \mathbb{Z}$ such that $120 n+3\left(s^{2}+t^{2}\right)+5=3 x^{2}+3 y^{2}+5 z^{2}$. Clearly, $x$ and $y$ cannot be both even. Without loss of generality, we assume that $2 \nmid x$. Then $3 y^{2}+5 z^{2}>0$ and $3 y^{2}+5 z^{2} \equiv 0$ $(\bmod 8)$. By Lemma 5.1, we can write $3 y^{2}+5 z^{2}$ as $3 y_{0}^{2}+5 z_{0}^{2}$ with $y_{0}$ and $z_{0}$ both odd. So, without loss of generality we may simply assume that $y$ and $z$ are also odd.

If $\{s, t\}=\{1,3\}$, then $\psi_{5, s}(y)+\psi_{5, t}(z) \sim T_{y}+T_{z}$ by $(1.10)$, hence $\psi_{3,1}(x)+\psi_{5, s}(y)+$ $\psi_{5, t}(z)$ is universal over $\mathbb{Z}$ as $p_{5}(x)+T_{y}+T_{z}$ is universal over $\mathbb{Z}$ by [S15, Theorem 1.14].

Now we assume that $\{s, t\} \neq\{1,3\}$. Clearly, $z$ or $-z$ has the form $6 w+1$ with $w \in \mathbb{Z}$. Since $x^{2}+y^{2} \equiv s^{2}+t^{2} \not \equiv 0(\bmod 5)$, we have $x^{2}+y^{2}=(10 u+s)^{2}+(10 v+t)^{2}$ for some $u, v \in \mathbb{Z}$. Therefore

$$
120 n+3 s^{2}+3 t^{2}+5=5(6 w+1)^{2}+3(10 u+s)^{2}+3(10 v+t)^{2}
$$

and hence $n=w(3 w+1) / 2+u(5 u+s) / 2+v(5 v+t) / 2$. This proves $(1.15)$.

In view of the above, we have completed the proof of Theorem 1.11.

Acknowledgments. The author would like to thank the two referees and his graduate student Hai-Liang Wu for helpful comments.

\section{REFERENCES}

[A] S. Arno, The imaginary quadratic fields of class number 4, Acta Arith. 60 (1992), 321-334.

[ARW] S. Arno, M.L. Robinson, F.S. Wheeler, Imaginary quadratic fields with small odd class number, Acta Arith. 83 (1998), 295-330.

[B] B. C. Berndt, Number Theory in the Spirit of Ramanujan, Amer. Math. Soc., Providence, RI, 2006.

[C] J.W.S. Cassels, Rational Quadratic Forms, Dover Publ., Inc., New York, 1978.

[CO] W. K. Chan and B.-K. Oh, Representations of integral quadratic polynomials, in: Diophantine Methods, Lattices, and Arithmetic Theory of Quadratic Forms, pp. 31-46, Contemp. Math., 587, Amer. Math. Soc., Providence, RI, 2013.

[CL] S. Cooper and H. Y. Lam, On the diophantine equation $n^{2}=x^{2}+b y^{2}+c z^{2}$, J. Number Theory 133 (2013), 719-737.

[D39] L. E. Dickson, Modern Elementary Theory of Numbers, University of Chicago Press, Chicago, 1939.

[D99] L. E. Dickson, History of the Theory of Numbers, Vol. II, AMS Chelsea Publ., 1999.

[DW] G. Doyle and K. S. Williams, A positive-definite ternary quadratic form does not represent all positive integers, Integers 17 (2017), \#A41, pp. 1-19 (eletronic).

[GS] F. Ge and Z.-W. Sun, On some universal sums of generalized polygonal numbers, Colloq. Math. 145 (2016), 149-155.

[GPS] S. Guo, H. Pan and Z.-W. Sun, Mixed sums of squares and triangular numbers (II), Integers 7 (2007), \#A56, 5pp (electronic).

[G94] R. K. Guy, Every number is expressible as the sum of how many polygonal numbers?, Amer. Math. Monthly 101 (1994), 169-172. 
[JKS] W. C. Jagy, I. Kaplansky and A. Schiemann, There are 913 regular ternary forms, Mathematika 44 (1997), 332-341.

[JP] B. W. Jones and G. Pall, Regular and semi-regular positive ternary quadratic forms, Acta Math. 70 (1939), 165-191.

[JO] J. Ju and B.-K. Oh, A generalization of Gauss' triangular theorem, Bull. Korean Math. Soc. 55 (2018), 1149-1159.

[JOS] J. Ju, B.-K. Oh and B. Seo, Ternary universal sums of generalized polygonal numbers, preprint, arXiv: 1612.01157, 2016.

[N96] M. B. Nathanson, Additive Number Theory: The Classical Bases, Grad. Texts in Math., Vol. 164, Springer, New York, 1996.

[O11] B.-K. Oh, Ternary universal sums of generalized pentagonal numbers, J. Korean Math. Soc. 48 (2011), 837-847.

[OS] B.-K. Oh and Z.-W. Sun, Mixed sums of squares and triangular numbers (III), J. Number Theory 129 (2009), 964-969.

[P] P. Pollack, Not Always Buried Deep - A Second Course in Elementary Number Theory, Amer. math. Soc., Providence, RI, 2009.

[S07] Z.-W. Sun, Mixed sums of squares and triangular numbers, Acta Arith. 127 (2007), 103-113.

[S15] Z.-W. Sun, On universal sums of polygonal numbers, Sci. China Math. 58 (2015), 1367-1396.

[S17] Z.-W. Sun, On $x(a x+1)+y(b y+1)+z(c z+1)$ and $x(a x+b)+y(a y+c)+z(a z+d)$, J. Number Theory 171 (2017), 275-283.

[S17a] Z.-W. Sun, Sequence A286944 in OEIS (On-Line Encyclopedia of Integer Sequences), available from http://oeis.org/A286944, 2017.

[W] C. Wagner, Class number 5, 6 and 7, Math. Comp. 65 (1996), 785-800.

[Wa] M. Watkins, Class numbers of imaginary quadratic fields, Math. Comp. 73 (2004), 907-938.

[We] E. Weisstein, Class Number from MathWorld, http://mathworld.wolfram/ClassNumber.html. 


\section{ApPENDIX}

In this appendix, we list our conjectural universal tuples $(a, b, c, d, e, f)$ over $\mathbb{N}$ with $a \geqslant c \geqslant e \geqslant 1, b>-a$ and $b \equiv a(\bmod 2), d>-c$ and $d \equiv c(\bmod 2), f>-e$ and $f \equiv e(\bmod 2)$, and $(a \nmid b$ or $c \nmid d$ or $e \nmid f)$. For each of the listed tuple $(a, b, c, d, e, f)$, we have verified that every $n=0, \ldots, 10^{6}$ can be written as $\psi_{a, b}(x)+\psi_{c, d}(y)+\psi_{e, f}(z)$ with $x, y, z \in \mathbb{N}$. Below is our list.

$$
\begin{aligned}
& (3,-1,1,1,1,1),(3,-1,1,3,1,1),(3,-1,1,5,1,1),(3,-1,1,7,1,1), \\
& (3,-1,1,9,1,1),(3,-1,1,11,1,1),(3,-1,1,13,1,1),(3,-1,1,15,1,1), \\
& (3,-1,1,17,1,1),(3,-1,2,0,1,1),(3,-1,2,0,1,3),(3,-1,2,0,1,5), \\
& (3,-1,2,0,2,0),(3,-1,2,2,1,1),(3,-1,2,2,1,3),(3,-1,2,2,2,0), \\
& (3,-1,2,4,1,1),(3,-1,2,4,1,3),(3,-1,2,4,2,2),(3,-1,2,6,1,1), \\
& (3,-1,2,8,1,1),(3,-1,2,10,1,1),(3,-1,2,12,1,1),(3,-1,2,16,1,1), \\
& (3,-1,3,-1,1,1),(3,-1,3,-1,1,3),(3,1,1,1,1,1),(3,1,1,3,1,1), \\
& (3,1,1,5,1,1),(3,1,1,7,1,1),(3,1,2,0,1,1),(3,1,2,0,1,3), \\
& (3,1,2,2,1,1),(3,1,2,2,2,0),(3,1,2,4,1,1),(3,1,2,6,1,1), \\
& (3,1,2,6,2,0),(3,1,3,-1,1,1),(3,1,3,-1,1,3),(3,1,3,-1,2,2), \\
& (3,1,3,-1,2,6),(3,3,3,-1,1,1),(3,3,3,-1,1,3),(3,3,3,-1,2,0), \\
& (3,3,3,-1,2,2),(3,3,3,1,1,1),(3,3,3,1,2,0),(3,5,1,1,1,1), \\
& (3,5,1,3,1,1),(3,5,2,0,1,1),(3,5,2,0,1,3),(3,5,2,2,1,1), \\
& (3,5,2,2,2,0),(3,5,3,-1,1,1),(3,5,3,1,1,1),(3,7,1,1,1,1), \\
& (3,7,2,0,1,1),(3,7,2,2,2,0),(3,7,3,-1,1,1),(3,9,3,-1,1,1), \\
& (3,11,2,0,1,1),(3,11,2,0,1,3),(3,11,3,-1,1,1),(3,13,2,0,1,1), \\
& (3,15,3,-1,1,1) ;
\end{aligned}
$$

$$
\begin{aligned}
& (4,-2,1,1,1,1),(4,-2,1,3,1,1),(4,-2,1,3,1,3),(4,-2,1,5,1,1), \\
& (4,-2,1,5,1,3),(4,-2,1,7,1,1),(4,-2,1,7,1,3),(4,-2,1,9,1,1), \\
& (4,-2,2,0,1,1),(4,-2,2,0,1,3),(4,-2,2,0,1,5),(4,-2,2,2,1,1), \\
& (4,-2,2,2,1,3),(4,-2,2,2,1,5),(4,-2,2,2,2,0),(4,-2,2,4,1,1), \\
& (4,-2,2,8,1,1),(4,-2,3,-1,1,1),(4,-2,3,-1,1,3),(4,-2,3,-1,1,5), \\
& (4,-2,3,-1,2,0),(4,-2,3,1,1,1),(4,-2,3,1,1,3),(4,-2,3,1,2,0), \\
& (4,-2,3,7,1,1),(4,0,3,-1,1,1),(4,0,3,-1,1,3),(4,0,3,-1,1,5), \\
& (4,0,3,-1,1,7),(4,0,3,-1,2,0),(4,0,3,-1,2,4),(4,0,3,1,1,1), \\
& (4,0,3,1,2,0),(4,0,3,5,1,1),(4,0,3,5,2,0),(4,0,4,-2,1,1) . \\
& (4,0,4,-2,1,3),(4,0,4,-2,1,5),(4,0,4,-2,3,-1),(4,2,2,0,1,1) . \\
& (4,2,2,0,1,3),(4,2,3,-1,1,1),(4,2,3,-1,1,3),(4,4,3,-1,1,1), \\
& (4,4,3,1,1,1),(4,6,3,-1,1,1),(4,8,3,-1,1,1),(4,10,3,-1,1,1)
\end{aligned}
$$


$(5,-3,1,1,1,1),(5,-3,1,3,1,1),(5,-3,1,5,1,1),(5,-3,1,7,1,1)$, $(5,-3,1,9,1,1),(5,-3,2,0,1,1),(5,-3,2,0,1,3),(5,-3,2,2,1,1)$, $(5,-3,2,2,1,3),(5,-3,2,2,2,0),(5,-3,2,4,1,1),(5,-3,2,4,1,3)$, $(5,-3,2,4,2,2),(5,-3,2,8,1,1),(5,-3,3,-1,1,1),(5,-3,3,1,1,1)$, $(5,-3,3,1,1,3),(5,-3,3,3,3,-1),(5,-3,3,7,1,1),(5,-1,2,0,1,1)$, $(5,-1,2,0,1,3),(5,-1,2,0,1,5),(5,-1,2,0,1,7),(5,-1,2,0,1,9)$, $(5,-1,2,2,1,1),(5,-1,2,6,1,1),(5,-1,3,-1,1,1),(5,-1,3,1,1,1)$, $(5,-1,4,0,1,1),(5,1,2,0,1,1),(5,1,2,0,1,3),(5,1,2,2,1,1)$, $(5,1,3,-1,1,1),(5,1,3,-1,1,3),(5,1,3,1,1,1),(5,3,1,1,1,1)$, $(5,3,1,3,1,1),(5,3,2,0,1,1),(5,3,2,2,1,1),(5,3,2,2,2,0)$, $(5,3,3,-1,1,1),(5,3,3,1,2,0),(5,3,4,-2,1,1),(5,3,4,-2,1,3)$, $(5,7,2,0,1,1),(5,7,2,0,1,3),(5,7,3,-1,1,1),(5,9,3,-1,1,1)$, $(5,11,2,0,1,1),(5,13,3,-1,1,1)$;

$(6,-4,1,1,1,1),(6,-4,1,3,1,1),(6,-4,1,3,1,3),(6,-4,1,5,1,1)$, $(6,-4,1,5,1,3),(6,-4,1,7,1,1),(6,-4,1,7,1,3),(6,-4,1,9,1,1)$, $(6,-4,2,0,1,1),(6,-4,2,0,1,3),(6,-4,2,2,1,1),(6,-4,2,2,1,3)$, $(6,-4,2,2,2,0), \quad(6,-4,2,6,1,1),(6,-4,2,6,1,3),(6,-4,3,-1,1,1)$, $(6,-4,3,1,1,1),(6,-4,3,1,1,3),(6,-4,3,1,1,5),(6,-4,3,5,1,1)$, $(6,-4,3,7,1,1),(6,-4,4,0,1,1),(6,-4,4,0,1,3),(6,-4,4,0,1,5)$, $(6,-4,4,0,1,7),(6,-4,5,-3,1,1),(6,-2,1,1,1,1),(6,-2,1,3,1,1)$, $(6,-2,1,5,1,1),(6,-2,1,7,1,1),(6,-2,2,0,1,1),(6,-2,2,0,1,3)$, $(6,-2,2,0,1,5),(6,-2,2,0,1,7),(6,-2,2,0,1,9),(6,-2,2,2,2,0)$, $(6,-2,3,1,1,1),(6,-2,4,-2,1,1),(6,-2,4,-2,1,3),(6,-2,4,-2,1,5)$, $(6,-2,4,0,1,1),(6,-2,4,4,1,1),(6,-2,5,-3,1,1),(6,-2,5,-3,1,3)$, $(6,0,3,-1,1,1),(6,0,3,-1,2,2),(6,0,3,1,1,1),(6,0,6,-4,1,1)$, $(6,0,6,-4,2,2),(6,2,1,1,1,1),(6,2,1,3,1,1),(6,2,2,2,1,1)$, $(6,2,2,2,2,0),(6,2,3,-1,1,1),(6,2,3,1,1,1),(6,2,4,0,1,1)$, $(6,2,6,-4,1,1),(6,4,1,1,1,1),(6,4,2,2,2,0),(6,4,6,-4,1,1)$, $(6,6,3,-1,1,1),(6,8,2,0,1,1),(6,8,3,-1,1,1),(6,10,2,0,1,1)$, $(6,12,3,-1,1,1)$; 
$(7,-5,2,0,1,1),(7,-5,2,2,1,1),(7,-5,2,4,1,1),(7,-5,2,6,1,1)$, $(7,-5,2,8,1,1),(7,-5,3,-1,1,1),(7,-5,3,-1,1,3),(7,-5,3,-1,2,2)$, $(7,-5,3,5,1,1),(7,-5,4,0,1,1),(7,-3,2,0,1,1),(7,-3,2,2,1,1)$, $(7,-3,2,6,1,1),(7,-3,3,-1,1,1),(7,-3,3,-1,1,3),(7,-3,3,-1,1,5)$, $(7,-3,3,1,1,1),(7,-3,4,0,1,1),(7,-1,2,0,1,1),(7,-1,2,0,1,3)$, $(7,-1,2,2,1,1),(7,-1,3,1,1,1),(7,-1,6,-4,1,1),(7,1,2,0,1,1)$, $(7,1,3,-1,1,1),(7,3,1,1,1,1),(7,3,2,2,2,0),(7,5,2,0,1,1)$, $(7,5,2,0,1,3)$;

$(8,-6,1,1,1,1),(8,-6,1,3,1,1),(8,-6,1,5,1,1),(8,-6,1,7,1,1)$, $(8,-6,2,0,1,1),(8,-6,2,2,1,1),(8,-6,2,2,1,3),(8,-6,2,2,2,0)$, $(8,-6,2,4,2,2),(8,-6,3,1,1,1),(8,-6,5,-3,1,1),(8,-6,5,-3,1,3)$, $(8,-4,2,2,1,1),(8,-4,2,6,1,1),(8,-4,6,-4,1,1),(8,-2,3,-1,1,1)$, $(8,-2,3,1,1,1),(8,0,3,-1,1,1),(8,0,3,1,1,1),(8,0,6,-2,1,1)$, $(8,2,1,1,1,1),(8,2,2,2,2,0)$;

$(9,-7,2,0,1,1),(9,-7,2,4,1,1),(9,-7,2,8,1,1),(9,-7,3,-1,1,1)$, $(9,-7,5,-1,1,1),(9,-7,5,-1,1,3),(9,-5,2,2,1,1),(9,-5,2,6,1,1)$, $(9,-5,3,-1,1,1),(9,-5,3,-1,1,3),(9,-5,3,5,1,1),(9,-5,4,0,1,1)$, $(9,-1,1,1,1,1),(9,-1,1,3,1,1),(9,-1,2,0,1,1),(9,-1,2,2,1,1)$, $(9,-1,2,2,2,0),(9,-1,4,0,1,1),(9,5,3,-1,1,1),(9,7,2,0,1,1)$, $(9,9,3,-1,1,1)$;

$(10,-8,1,1,1,1),(10,-8,1,3,1,1),(10,-8,1,5,1,1),(10,-8,1,7,1,1)$, $(10,-8,1,9,1,1),(10,-8,2,0,1,1),(10,-8,2,2,1,1),(10,-8,2,2,1,3)$, $(10,-8,2,2,2,0),(10,-8,2,4,1,1),(10,-8,2,8,1,1),(10,-8,3,1,1,1)$, $(10,-8,3,1,1,3), \quad(10,-8,3,7,1,1),(10,-6,2,0,1,1),(10,-6,2,0,1,3)$, $(10,-6,2,0,1,5),(10,-6,2,2,1,1),(10,-6,3,-1,1,1),(10,-6,3,5,1,1)$, $(10,-6,5,-3,1,1),(10,-4,2,0,1,1),(10,-4,2,0,1,3),(10,-4,3,-1,1,1)$, $(10,-2,3,-1,1,1),(10,2,3,-1,1,1),(10,4,3,-1,1,1),(10,6,2,0,1,1)$, $(10,8,3,-1,1,1)$;

$(11,-9,2,0,1,1),(11,-9,3,-1,1,1),(11,-9,3,1,1,1),(11,-9,3,7,1,1)$, $(11,-7,1,1,1,1),(11,-7,1,3,1,1),(11,-7,1,5,1,1),(11,-7,2,2,2,0)$, $(11,-7,3,-1,1,1),(11,-5,2,0,1,1),(11,-5,2,0,1,3),(11,-3,3,-1,1,1)$, $(11,-3,3,1,1,1),(11,5,2,0,1,1)$; 
$(12,-8,2,0,1,1),(12,-8,2,0,1,3),(12,-8,2,2,1,1),(12,-4,1,1,1,1)$, $(12,-4,1,3,1,1),(12,-4,2,2,1,1),(12,-4,2,2,2,0),(12,-4,4,0,1,1)$. $(13,-11,2,0,1,1),(13,-11,2,2,1,1),(13,-11,2,2,1,3),(13,-9,2,0,1,1)$, $(13,-9,2,0,1,3),(13,-7,2,0,1,1),(13,-7,2,0,1,3),(13,-5,2,0,1,1)$. $(14,-12,2,2,1,1),(14,-10,2,0,1,1),(14,-10,2,0,1,3),(14,-10,2,2,1,1)$, $(14,-10,3,-1,1,1),(14,-2,2,0,1,1),(14,-2,3,-1,1,1),(14,2,2,0,1,1)$, $(15,-13,1,1,1,1),(15,-13,1,3,1,1),(15,-13,1,5,1,1),(15,-13,1,7,1,1)$, $(15,-13,1,9,1,1),(15,-13,2,0,1,1),(15,-13,2,2,1,1),(15,-13,2,2,1,3)$, $(15,-13,2,2,2,0),(15,-13,2,4,1,1),(15,-13,2,4,2,2),(15,-13,2,8,1,1)$, $(15,-13,3,1,1,1),(15,-13,3,7,1,1),(15,-13,4,0,1,1),(15,-11,1,1,1,1)$, $(15,-11,2,0,1,1),(15,-11,2,2,2,0),(15,-7,1,1,1,1),(15,-7,2,0,1,1)$, $(15,-7,2,2,1,1),(15,-7,2,2,2,0),(15,-3,3,-1,1,1),(15,3,3,-1,1,1)$, $(16,-14,2,0,1,1),(16,-10,2,0,1,1),(16,-10,2,0,1,3),(16,-8,3,-1,1,1)$, $(16,-4,2,0,1,1),(17,-15,3,1,1,1),(17,-15,3,1,1,3),(18,-10,2,0,1,1)$, $(20,-16,2,2,1,1),(20,-16,2,6,1,1),(20,-12,3,-1,1,1),(20,-4,2,0,1,1)$, $(21,-19,2,2,1,1),(21,-9,2,0,1,1),(21,-5,2,0,1,1),(25,-23,2,0,1,1)$. 